\title{
Acupuncture for Cancer-Induced Bone Pain in Animal Models: A Systemic Review and Meta-Analysis
}

\author{
Mei-ling Yu, ${ }^{1,2}$ Jia-jia Qian, ${ }^{1,2}$ Shu-ping Fu ${ }^{(D},{ }^{3}$ Jia-ying Chen, ${ }^{2}$ Yu-wen Zheng, ${ }^{2}$ \\ Zhi-gang $L u\left(\mathbb{1},,^{1,4}\right.$ and Sheng-feng $L u \mathbb{1}^{3}$ \\ ${ }^{1}$ First Clinical Medical College, Nanjing University of Chinese Medicine, Nanjing 210023, China \\ ${ }^{2}$ Acupuncture and Rehabilitation College, Nanjing University of Chinese Medicine, Nanjing 210023, China \\ ${ }^{3}$ Key Laboratory of Acupuncture and Medicine Research of Ministry of Education, Nanjing University of Chinese Medicine, \\ Nanjing 210023, China \\ ${ }^{4}$ College of Pharmacy, Nanjing University of Chinese Medicine, Nanjing 210023, China
}

Correspondence should be addressed to Zhi-gang Lu; Luzg@njucm.edu.cn and Sheng-feng Lu; lushengfeng@njucm.edu.cn

Received 2 February 2020; Revised 11 June 2020; Accepted 2 July 2020; Published 30 July 2020

Academic Editor: Vincenzo De Feo

Copyright (c) 2020 Mei-ling Yu et al. This is an open access article distributed under the Creative Commons Attribution License, which permits unrestricted use, distribution, and reproduction in any medium, provided the original work is properly cited.

\begin{abstract}
Background. Cancer-induced bone pain (CIBP) is a highly prevalent symptom, which afflicts vast majority of patients who suffer from cancer. The current treatment options failed to achieve satisfactory effect and the side effects were prominent. Recent randomized controlled trials (RCTs) of animal demonstrate the benefit of acupuncture for CIBP. We sought to determine if the pooled data from available RCTs supports the use of acupuncture for CIBP. Methods. A literature search for randomized controlled trials was conducted in six electronic databases from inception to May 31, 2019. Meta-analysis was performed with Review Manager 5.3 software; the publication bias was assessed by Stata 12.0 software. We used random effects model for pooling data because heterogeneity is absolute among studies to some extent. Results. Twenty-four trials were included in the review, of which 12 trials provided detailed data for meta-analyses. Preliminary evidence indicates that compared to wait list/sham group, acupuncture was effective on increasing paw withdrawal threshold (PWT) and paw withdrawal latency (PWL). Compared to medicine, acupuncture was less effective on PWT, but as effective as medicine on PWL. Acupuncture can reinforce medicine's effect on PWT and PWL. Compared to the control group, acupuncture was superior to increase body weight (BW), decrease spinal cord glial fibrillary acidic protein (GFAP), and interleukin-1 $\beta$ (IL-1 $\beta$ ). Furthermore, some studies showed acupuncture delay or partially reverse morphine tolerance. Three studies found acupuncture has no effect on PWT, but 2 of them found acupuncture could enhance small dose of Celebrex's effect on CIBP. Conclusions. Acupuncture was superior to wait list/sham acupuncture on increasing PWT and has no less effect on increasing PWL compared to medicine; acupuncture improved the efficacy of drugs, increased the CIBP animals' body weight, and decreased their spinal cord GFAP and IL-1 $\beta$. High-quality studies are necessary to confirm the results.
\end{abstract}

\section{Introduction}

Cancer-induced bone pain (CIBP) refers to bone pain caused by primary bone neoplasm or secondary to other carcinomas. It is a highly prevalent symptom, which afflicts vast majority of patients who suffer from cancer [1]. Primary bone cancers just account for less than $1 \%$ of diagnosed cancers every year [2]; bone metastasis, which greatly increases patient's risk of fractures and other structural complications, is the most common contributor of cancer- related pain [3]. Bone pain limits patient's mobility; increases depression and anxiety; and increases risk of lung infection, cutaneous ulcers, and vein thrombosis [4]. Effective treatment of CIBP is urgent as the patients' survival increases due to rapid development of new available target treatments.

Up to now, the three-step ladder World Health Organization (WHO) model is still the most frequently used program for cancer pain clinically. Opioids remain considered as one of the most effective pain-relieving 
therapeutics. Oral morphine is the first choice in patients who can assume oral drugs [5]. However, the long-term use of opioids is limited by the development of tolerance, potential for addiction and hyperalgesia [6-8]. Besides, up to $40 \%$ of cancer patients with no previous emesis complained of opioid-induced nausea and vomiting; meanwhile, opioidrelated constipation and center nervous system (CNS) symptoms deserve attention [5]. It should be notice that, compared with other pain states, the analgesic effects of opioids can be less effective in CIBP [9-11]. Evidence indicates that at least nearly $25.5 \%-31 \%$ of patients have been troubled by severe pain and do not get satisfactory treatment, while undertreatment of cancer pain was negatively associated with patients' overall well-being [12-14]. Furthermore, about $70 \%$ of patients reported side events associated with their pain medication, which impose negative impact on activities of daily living [13]. Based on the above reasons, patients and clinicians aspire to better treatment with definite effects and fewer side effects.

As one of the traditional Chinese medicines, acupuncture is known for its effect on pain-relieving in cancer therapy and almost free of adverse effect. Plenty of studies have proven that acupuncture is effective on some cancer-induced pain, such as pain arising from hepatocellular carcinoma [15], stomach carcinoma [16], ovarian cancer [17], pancreatic cancer [18], breast cancer [19], and so on. Can acupuncture be used to alleviate CIBP? Clinical study in this area is rare, but it is worth to notice that animal research is gradually increasing. To clarify the potential benefits of acupuncture for CIBP in animals, we conducted this systematic review and meta-analysis. The review protocol was registered with PROSPERO (CRD42019133175).

\section{Material and Methods}

2.1. Inclusion and Exclusion Criteria. We carried out the systematic review following Cochrane and preferred reporting items for systematic reviews and meta-analysis (PRISMA) guidelines. The studies were included if they explored acupuncture's effect on cancer-induced bone pain. Stimulation on acupoint, whether using needle insertion or other mediators, was accepted. Different types of acupuncture comparison were excluded. Randomized controlled trials were eligible for inclusion. Studies were included in the analysis more than once if they had different arms. If more than one scheme was employed, only the most effective was used. The primary measures of treatment benefit were PWT and PWL. BW (body weight), spinal cord glial fibrillary acidic protein (GFAP), and interleukin- $\beta$ (IL- $\beta$ ) were chosen as the secondary outcomes. Studies that did not include the primary or secondary outcomes were excluded. The primary exclusion criteria were observational nonrandomized studies, ongoing trials without results, case reports, reviews, and duplicate studies. Studies that did not include a usual-care and/or placebo comparison group and for which a final data analysis was not available were excluded.
2.2. Search Strategy. Briefly, we searched PubMed, Embase, China National Knowledge Infrastructure (CNKI), Chinese Science and Technology Periodical Database (VIP), Wanfang database, and China Biomedical Literature Service System (SinoMed). Literature searches were conducted from their inception up to May 31, 2019. MeSH terms for PubMed and comparable terms for other databases were employed during search. The following search terms were included in various combinations: "manual acupuncture"/"electroacupuncture(EA)"/"five-element acupuncture"/"auricular acupuncture"/"auricular needle"/"laser needle"/"transcutaneous acupoint electrical stimulation"/"catgut implantation", and "cancer-induced bone pain"/"bone tumor"/"bone marrow cancer"/“multiple myeloma"/"bone neoplasm"/ "osseous metastasis"/"bone metastasis"/"skeletal metastases"/“bone cancer". All timings, frequencies, and durations of treatment are eligible for inclusion. Bibliographies of relevant papers were screened. Searches were limited to animal studies published in English and Chinese. Case reports, editorials, letters, comments, or any types of reviews were excluded.

2.3. Data Screening and Extraction. All studies that met the inclusion criteria were arranged to data screen and extract. Two reviewers (Mei-ling Yu and Jia-jia Qian) screened titles and abstracts for initial inclusion in an independent manner, and disagreements were resolved by discussion and reviewing the full paper. Two reviewers (Jia-ying Chen and Yu-wen Zheng) performed data extraction on the included trials, and disagreements were settled by consulting a third reviewer (Mei-ling Yu).

2.4. Evaluation of Risk of Bias. Study quality was evaluated with Systematic Review Centre for Laboratory Animal Experimentation (SYRCLE) and Standard for Reporting Interventions in Clinical Trials of Acupuncture (STRICTA) by independent investigators (Sheng-feng $\mathrm{Lu}$ and Jia-ying Chen). We used the SYRCLE's risk of bias (RoB) tool to assess risk of bias of the included literature. The following contents are contained [20]: (1) selection bias; (2) performance bias; (3) detection bias; (4) attrition bias; (5) reporting bias; and (6) other sources of bias. The STRICTA checklist was employed to assess the quality of acupuncture trials. The following contents are contained [21]: (1) acupuncture rationale; (2) details of needling; (3) treatment regimen; (4) other components of treatment; (5) practitioner background; and (6) control or comparator interventions.

2.5. Statistical Analysis. Given that heterogeneity is inevitable across studies, random effects meta-analysis was used to synthesize results. $I^{2}$ was employed to quantify the heterogeneity $\left(I^{2}<25 \%\right.$-low, $25-50 \%$ - moderate, and $>50 \%-$ high degree of heterogeneity). We conducted sensitivity analyses to explore the robustness of our results and to evaluate whether any of the included studies had a large influence on the results. Data were summarized by standard mean difference (SMD) with 95\% CI for continuous 
outcomes. A $\chi^{2}$-based test of homogeneity was performed and the inconsistency index $\left(I^{2}\right)$ and $Q$ statistics were determined. Pooled effects were calculated and a 2 -sided $P$ value $<0.05$ was considered to indicate statistical significance. The leave-one-out approach was used for sensitivity analysis of the primary outcome. Publication bias was assessed by funnel plot and egger's test. Analysis was conducted in Review Manager 5.3 and Stata 12.0 software.

\section{Results}

3.1. Study Selection. A total of 723 articles were identified with the search terms. 82 duplicates were omitted, and the left 641 publications were screened. 564 studies were excluded for at least one of the following reasons after title and abstract were screened: (1) the intervention did not include acupuncture; (2) the stimulation site was not at the acupoint; (3) the model was not cancer-induced bone pain; (4) they were not animal studies; (5) they were reviews or case reports or letters; (6) the outcomes did not include PWT/PWL/BW/ GFAP/IL- $1 \beta$; and (7) they did not include a usual-care and/ or placebo comparison group. 77 articles remained after initial reading. Then, we read the full papers: 53 papers were excluded and 24 articles met the inclusion criteria finally (Figure 1). Among them, 3 papers were from Ph.D. dissertations. By reading the full texts of the included studies, we found some studies investigating acupuncture effects from various aspects, so they were divided into more than one RCT according to their arms. Finally, the meta-analysis was divided into 3 parts: (1) acupuncture with control; (2) acupuncture with western medicine; and (3) combination of acupuncture and medication with medication.

3.2. Study Characteristics. The detailed information of the animal model is provided in Table 1. 977 animals were involved totally. 12 studies used female SD rats; 3 studies used male SD rats; 6 studies used female Wistar rats; male Copenhagen rat was employed in 2 studies; and $\mathrm{BALB} / \mathrm{c}$ mice were used in 1 study. 19 studies used walker256 mammary gland carcinoma cells to made model; 2 studies used MRMT-1 mammary gland carcinoma cells; 2 studies used AT 3.1 prostate cancer cell line; and 1 study used K7M2 osteosarcoma cell strain.

The acupuncture treatment parameters of the included studies are listed in Table 2. Among all the studies, a total of 8 points involving 4 channels, as well as the extra points, were used. The frequencies of acupuncture points from high to low were as follows: ST36, 19 times; BL60, 12 times; GB30, 4 times; L3-L5 EX-B2, 3 times; BL2, 2 times; SP6, 2 times; and GenDuan, 2 times. Needle retaining was for $30 \mathrm{~min}$ in 22 studies, $6.5 \mathrm{~min}$ in 1 study, and $13 \mathrm{~min}$ in 1 study. All studies used electroacupuncture (EA). The electrical frequency employed was 2/ $100 \mathrm{~Hz}$ in 15 studies; the rest frequency was decrement in turn: $15 \mathrm{~Hz}, 4 \mathrm{~Hz}, 10 \mathrm{~Hz}, 2 \mathrm{~Hz}$, and $4 / 60 \mathrm{~Hz}$. One study did not report the EA frequency. EA intensity of all the included studies was between 0.3 and $2.0 \mathrm{~mA}$ except for Li2010 [24]. The frequency of EA treatment was as follows: 15 studies provided acupuncture once every day; 7 studies provided acupuncture every other day;

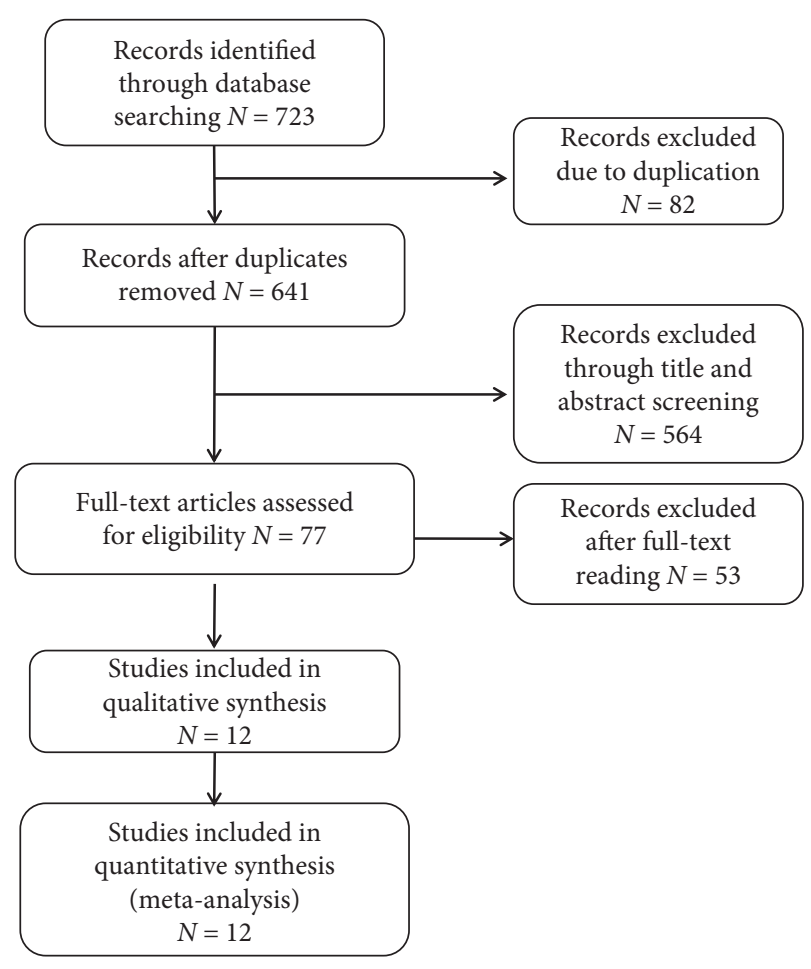

Figure 1: Flow diagram for study selection.

1 study provided acupuncture 2 times per week; and 1 study was investigate the effect of continuum and interval treatment. The number of treatments ranged from 5 to 20 times, mainly focusing on 6 and 7 times. No serious adverse events have been reported.

3.3. Quality Assessment. Standard for Reporting Interventions in Clinical Trials of Acupuncture (STRICTA) [44] checklist was used to assess the study quality from the aspect of acupuncture intervention (Table 3 ). Of the 24 included studies, all studies provided detailed style of acupuncture, 23 provided the detailed reasoning for treatment, and all studies described the extent to which treatment was varied. 22 studies provide the number of needle insertions per subject per session and the names of points used. 14 studies described the depth of insertion. Seven studies described the response sought. All the included studies used electrical stimulation during needle retention. All the studies described the needle retention time. Just 1 study described the needle type, 11 studies did not provide detailed information, and the remaining 12 studies did not provide any information of the needle type. All the included studies described the number of treatment sessions, frequency, and duration of treatment sessions. All the included studies described the details of other interventions administered to the acupuncture group. None of the included studies described the setting and context of treatment, participating acupuncturists, rationale for the control, or comparator in the context of the research question, with sources that justify this choice. All the included studies provided precise description of the control or comparator.

Systematic Review Centre for Laboratory Animal Experimentation (SYRCLE) [45] risk of bias tool was applied to assess 
TABLE 1: Detailed information of animal models included in the study.

\begin{tabular}{|c|c|c|c|c|c|}
\hline Study ID & Objects & Cell strain & Carcinoma cell & Species & Inject location \\
\hline Qiliang [20] & Rat & Walker256 & MGCC & Wistar/F & Tibia \\
\hline Zhang et al [21] & Rat & AT-3.1 & PCC & Copenhagen/M & Tibia \\
\hline Qiliang et al. [22] & Rat & Walker256 & MGCC & Wistar/F & Tibia \\
\hline Zhang et al. [23] & Rat & AT-3.1 & PCC & Copenhagen/M & Tibia \\
\hline $\operatorname{Li}[24]$ & Rat & Walker256 & MGCC & Wistar/F & Tibia \\
\hline Smeester et al. [25] & Mouse & K7M2 & Osteosarcoma cell & $\mathrm{BALB} / \mathrm{c} 104$ female 166 male & Hind paw \\
\hline Kuai et al. [26] & Rat & Walker256 & MGCC & Wistar/F & Tibia \\
\hline Si-ma et al. [27] & Rat & Walker256 & MGCC & $\mathrm{SD} / \mathrm{F}$ & Tibia \\
\hline Zhao et al. [28] & Rat & Walker256 & MGCC & $\mathrm{SD} / \mathrm{F}$ & Tibia \\
\hline Zhao et al. [29] & Rat & Walker256 & MGCC & $\mathrm{SD} / \mathrm{F}$ & Tibia \\
\hline Du et al. [30] & Rat & Walker256 & MGCC & $\mathrm{SD} / \mathrm{F}$ & Tibia \\
\hline Du et al. [31] & Rat & Walker256 & MGCC & $\mathrm{SD} / \mathrm{F}$ & Tibia \\
\hline Lu et al. [32] & Rat & Walker256 & MGCC & Wistar/F & Tibia \\
\hline Lu et al. [33] & Rat & Walker256 & MGCC & Wistar/F & Tibia \\
\hline Sima et al. [34] & Rat & Walker256 & MGCC & $\mathrm{SD} / \mathrm{F}$ & Tibia \\
\hline Fu et al. [35] & Rat & Walker256 & MGCC & $\mathrm{SD} / \mathrm{F}$ & Tibia \\
\hline Fu et al. [36] & Rat & MRMT-1 & MGCC & $\mathrm{SD} / \mathrm{F}$ & Tibia \\
\hline Guan et al. [37] & Rat & Walker256 & MGCC & $\mathrm{SD} / \mathrm{M}$ & Tibia \\
\hline Liang et al. [38] & Rat & Walker 256 & MGCC & $\mathrm{SD} / \mathrm{F}$ & Tibia \\
\hline Liang et al. [39] & Rat & Walker256 & MGCC & $\mathrm{SD} / \mathrm{M}$ & Tibia \\
\hline Qin et al. [40] & Rat & Walker256 & MGCC & $\mathrm{SD} / \mathrm{M}$ & Tibia \\
\hline Cai et al. [41] & Rat & Walker256 & MGCC & $\mathrm{SD} / \mathrm{F}$ & Tibia \\
\hline Shi et al. [42] & Rat & MRMT-1 & MGCC & $\mathrm{SD} / \mathrm{F}$ & Tibia \\
\hline Guan et al. [43] & Rat & Walker256 & MGCC & $\mathrm{SD} / \mathrm{M}$ & Tibia \\
\hline
\end{tabular}

MGCC = mammary gland cancer cell; $\mathrm{PCC}=$ prostate cancer cell; $M=$ male; $\mathrm{F}=$ female.

TABLE 2: Characteristics of included studies.

\begin{tabular}{|c|c|c|c|c|c|c|c|c|}
\hline Study ID & Treatment/No. & Control/No. & Acupoints & Stimulator parameters & $\begin{array}{l}\text { No. of } \\
\text { treatments }\end{array}$ & Outcomes & $\begin{array}{l}\text { Side } \\
\text { effects }\end{array}$ & $P$ \\
\hline $\begin{array}{l}\text { Qiliang } \\
\text { [20] }\end{array}$ & $\begin{array}{c}\mathrm{EA}(12) \\
\mathrm{EA}+\operatorname{celecoxib}(12)\end{array}$ & $\begin{array}{c}\text { Model(10) } \\
\text { model + celecoxib(12) }\end{array}$ & $\begin{array}{l}\text { ST36, } \\
\text { BL60 }\end{array}$ & $\begin{array}{l}\mathrm{QID}, 4 / 60 \mathrm{HZ}, 1 \mathrm{~mA}, \\
30 \mathrm{~min} / \text { time }\end{array}$ & 10 & $\begin{array}{l}\text { PWT, PWL } \\
\text { BW }\end{array}$ & NR & $\begin{array}{l}P>0.05 \\
P<0.05\end{array}$ \\
\hline $\begin{array}{l}\text { Zhang } \\
\text { et al. [21] }\end{array}$ & $\mathrm{EA}(7)$ & $\operatorname{SEA}(7)$ & GB30 & $\begin{array}{l}\mathrm{QD}, 10 \mathrm{~Hz} / 2 \mathrm{~mA}, 30 \mathrm{~min} / \\
\text { time }\end{array}$ & 5 & PWLIL-1 $\beta$ & NR & $P<0.05$ \\
\hline $\begin{array}{l}\text { Zhang } \\
\text { et al. [23] }\end{array}$ & $\mathrm{EA}(7)$ & $\operatorname{SEA}(7)$ & GB30 & $\begin{array}{l}\mathrm{QD}, 10 \mathrm{~Hz} / 2 \mathrm{~mA}, 30 \mathrm{~min} / \\
\text { time }\end{array}$ & 5 & PWLPWPT & NR & $P<0.05$ \\
\hline $\begin{array}{l}\text { Qiliang } \\
\text { et al. [22] }\end{array}$ & $\begin{array}{c}\mathrm{EA}(12) \\
\mathrm{EA}+\text { celecoxib (12) }\end{array}$ & $\begin{array}{c}\text { Model(12) } \\
\text { Celecoxib(12) }\end{array}$ & $\begin{array}{l}\text { ST36, } \\
\text { BL60 }\end{array}$ & $\begin{array}{l}\mathrm{QD}, 4 / \mathrm{Hz}, 1 \mathrm{~mA}, 30 \mathrm{~min} / \\
\text { time }\end{array}$ & 20 & PWT & NR & $P<0.05$ \\
\hline $\operatorname{Li}[24]$ & $\begin{array}{c}\mathrm{EA}(16) \\
\mathrm{EA}\end{array}$ & $\begin{array}{l}\text { Model }(8) \\
\text { Model }\end{array}$ & $\begin{array}{c}\text { ST36/ } \\
\text { BL60 and } \\
\text { ST36/ } \\
\text { GB30 } \\
\text { ST36/ } \\
\text { GB30 }\end{array}$ & $\begin{array}{l}\text { QID, } 15 \mathrm{~Hz}, 2-5 \mathrm{~mA} \text {, } \\
30 \mathrm{~min} / \mathrm{time}, 15 \mathrm{~Hz}, 2- \\
5 \mathrm{~mA}, 30 \mathrm{~min} / \mathrm{time}\end{array}$ & 10 & PWT & NR & $P>0.05$ \\
\hline $\begin{array}{l}\text { Smeester } \\
\text { et al. [25] }\end{array}$ & $\mathrm{EA}(97)$ & $\begin{array}{l}\text { Model(39) } \\
\text { Sham EA(67) }\end{array}$ & ST36 & 2/week,4 Hz,30 min/time & 6 & PWT & NR & $P<0.01$ \\
\hline $\begin{array}{l}\text { Kuai et al. } \\
{[26]}\end{array}$ & $\mathrm{EA}(20)$ & $\begin{array}{c}\text { Model(10) } \\
\text { Morphine }(10)\end{array}$ & L3-L5 Jiaji & $\mathrm{QD}, 2 / 100 \mathrm{~Hz}, 30 \mathrm{~min} / \mathrm{time}$ & 6 & PWL & NR & $P<0.01$ \\
\hline $\begin{array}{l}\text { Zhao } \\
\text { et al. [28] }\end{array}$ & $\mathrm{EA}(10)$ & model(10) & BL2 & $\begin{array}{c}\mathrm{QD}, 2 / 100 \mathrm{~Hz} \\
0.3 \mathrm{~mA}, 13 \mathrm{~min} / \text { time }\end{array}$ & 12 & $\begin{array}{c}\text { PWT, } \\
\text { PWL, BW }\end{array}$ & NR & $P<0.05$ \\
\hline $\begin{array}{l}\text { Zhao } \\
\text { et al. [29] }\end{array}$ & $\begin{array}{c}\mathrm{EA}(8) \\
\mathrm{EA}(8) \\
\mathrm{EA}+\text { Zoledronic } \\
\operatorname{acid}(8)\end{array}$ & $\begin{array}{l}\text { Model }(8) \\
\text { Zoledronic } \operatorname{acid}(8) \\
\text { Zoledronic } \operatorname{acid}(8)\end{array}$ & BL2 & $\begin{array}{c}\mathrm{QD}, 2 / 100 \mathrm{~Hz}, 3 \mathrm{~mA}, \\
6.5 \mathrm{~min} / \mathrm{time}\end{array}$ & 12 & $\begin{array}{c}\text { BW, PWL } \\
\text { PWT }\end{array}$ & NR & $P<0.05$ \\
\hline $\begin{array}{l}\text { Si-ma } \\
\text { et al. [27] }\end{array}$ & $\mathrm{EA}(20)$ & Morphine(10) & ST36, SP6 & $\begin{array}{c}\mathrm{QD}, 2 / 100 \mathrm{~Hz}, 0.5-1.5 \mathrm{~mA}, \\
30 \mathrm{~min} / \mathrm{time}\end{array}$ & 9 & PWT & NR & $P<0.01$ \\
\hline $\begin{array}{l}\text { Du et al. } \\
\text { [30] }\end{array}$ & $\mathrm{EA}(42)$ & Model(7) & $\begin{array}{l}\text { ST36, } \\
\text { BL60 }\end{array}$ & $1-2 \mathrm{~mA}, 30 \mathrm{~min} /$ time & 7 & PWT & NR & $P<0.01$ \\
\hline
\end{tabular}


TABle 2: Continued.

\begin{tabular}{|c|c|c|c|c|c|c|c|c|}
\hline Study ID & Treatment/No. & Control/No. & Acupoints & Stimulator parameters & $\begin{array}{l}\text { No. of } \\
\text { treatments }\end{array}$ & Outcomes & $\begin{array}{l}\text { Side } \\
\text { effects }\end{array}$ & $P$ \\
\hline $\begin{array}{l}\text { Du et al. } \\
{[31]}\end{array}$ & $\mathrm{EA}(8)$ & Model (8) & $\begin{array}{c}\text { ST36, } \\
\text { GenDuan }\end{array}$ & $\begin{array}{c}\text { QID,2 Hz, } 1-2 \mathrm{~mA}, 30 \mathrm{~min} / \\
\text { time }\end{array}$ & 7 & PWT & NR & $P<0.05$ \\
\hline $\begin{array}{l}\text { Lu et al. } \\
{[32]}\end{array}$ & $\begin{array}{c}\mathrm{EA}(12) \\
\mathrm{EA}+\text { morphine }(12)\end{array}$ & $\begin{array}{c}\text { Model(12) } \\
\text { Morphine(12) }\end{array}$ & L3-5JiaJi & $\begin{array}{c}\mathrm{QD}, 2 / 100 \mathrm{~Hz}, 2 \mathrm{~mA}, \\
30 \mathrm{~min} / \mathrm{time}\end{array}$ & 6 & $\begin{array}{l}\text { PWL, } \\
\text { GFAP, IL- } \\
1 \beta\end{array}$ & NR & $P<0.01$ \\
\hline $\begin{array}{l}\text { Lu et al. } \\
\text { [33] }\end{array}$ & $\mathrm{EA}(8)$ & $\operatorname{Model}(8)$ & L3-5JiaJi & $\mathrm{QD}, 2 / 100 \mathrm{~Hz}, 30 \mathrm{~min} /$ time & 6 & PWL & NR & $P<0.05$ \\
\hline $\begin{array}{l}\text { Sima } \\
\text { et al. [34] }\end{array}$ & $\begin{array}{c}\mathrm{EA}(8) \\
\text { EA }+ \text { morphine } \\
\text { tolerance }(8)\end{array}$ & $\begin{array}{c}\text { SEA }(8) \\
\text { Model }(8) \\
\text { Morphine tolerance } \\
\text { Model }(8)\end{array}$ & ST36, SP6 & $\begin{array}{c}\mathrm{QD}, 2 / 100 \mathrm{~Hz}, 0.5-1.5 \mathrm{~mA} \\
30 \mathrm{~min} / \mathrm{time}\end{array}$ & 9 & PWT & NR & $P<0.01$ \\
\hline $\begin{array}{l}\text { Fu et al. } \\
{[35]}\end{array}$ & $\begin{array}{l}\text { EA I (8) } \\
\text { EA II (8) }\end{array}$ & $\begin{array}{l}\text { Morphine } \\
\text { tolerance }(8) \\
\text { SEA(11) }\end{array}$ & $\begin{array}{l}\text { ST36, } \\
\text { BL60 }\end{array}$ & $\begin{array}{l}\mathrm{QD}, 2 / 100 \mathrm{~Hz}, 2 / 100 \mathrm{~Hz} \\
0.5-1.5 \mathrm{~mA}, 30 \mathrm{~min} / \mathrm{time}\end{array}$ & $7 / 18$ & PWT & NR & $P<0.05$ \\
\hline $\begin{array}{l}\text { Fu et al. } \\
{[36]}\end{array}$ & $\mathrm{EA}(6)$ & $\begin{array}{l}\text { Model }(8) \\
\text { Morphine } \\
\text { tolerance }(8) \\
\text { SEA(6) }\end{array}$ & $\begin{array}{l}\text { ST36, } \\
\text { BL60 }\end{array}$ & $\begin{array}{c}\mathrm{QD}, 2 / 100 \mathrm{~Hz}, 0.5-1.5 \mathrm{~mA} \text {, } \\
30 \mathrm{~min} / \mathrm{time}\end{array}$ & 7 & PWT & NR & $P<0.01$ \\
\hline $\begin{array}{l}\text { Guan } \\
\text { et al. [37] }\end{array}$ & $\begin{array}{c}\mathrm{EA}+\text { herbal plaster } \\
(20)\end{array}$ & Herbal plaster(20) & $\begin{array}{l}\text { ST36, } \\
\text { BL60 }\end{array}$ & $\begin{array}{l}\mathrm{QID}, 2 / 100 \mathrm{~Hz}, 30 \mathrm{~min} / \\
\text { time, }\end{array}$ & 6 & $\begin{array}{c}\text { PWT, IL- } \\
1 \beta\end{array}$ & NR & $P<0.05$ \\
\hline $\begin{array}{l}\text { Liang } \\
\text { et al. [39] }\end{array}$ & $\mathrm{EA}(15)$ & $\begin{array}{c}\text { Model(13) } \\
\text { Morphine(13) }\end{array}$ & $\begin{array}{l}\text { ST36, } \\
\text { BL60 }\end{array}$ & $\begin{array}{l}\text { QID, 2/100 Hz, 0.5-1.0- } \\
1.5 \mathrm{~mA}, 30 \mathrm{~min} / \mathrm{time}\end{array}$ & 8 & PWT & NR & $P<0.01$ \\
\hline $\begin{array}{l}\text { Liang } \\
\text { et al. [38] }\end{array}$ & EA(13) & $\begin{array}{c}\text { Model(10) } \\
\text { Morphine(10) }\end{array}$ & $\begin{array}{l}\text { ST36, } \\
\text { BL60 }\end{array}$ & $\begin{array}{c}\text { QID, 2/100 Hz} \\
0.5-1.5 \mathrm{~mA}, 30 \mathrm{~min} / \text { time }\end{array}$ & 8 & PWT & NR & $P<0.01$ \\
\hline $\begin{array}{l}\text { Qin et al. } \\
{[40]}\end{array}$ & $\begin{array}{c}\mathrm{EA}(12) \\
\mathrm{EA}+\text { Herbal } \\
\text { plaster(12) }\end{array}$ & $\begin{array}{l}\text { Model(12) } \\
\text { plaster(12) }\end{array}$ & $\begin{array}{l}\text { ST36, } \\
\text { BL60 }\end{array}$ & $\begin{array}{c}\text { QD, 2/ } \\
100 \mathrm{~Hz}, 0.5-1.5 \mathrm{~mA} 30 \mathrm{~min} / \\
\text { time }\end{array}$ & 12 & PWL, PWT & NR & $P<0.01$ \\
\hline $\begin{array}{l}\text { Cai et al. } \\
{[41]}\end{array}$ & $\mathrm{EA}(13)$ & $\begin{array}{c}\text { Model(10) } \\
\text { Morphine(10) }\end{array}$ & $\begin{array}{l}\text { ST-36, } \\
\text { BL60 }\end{array}$ & $\begin{array}{c}\text { QID, } 2 / \\
100 \mathrm{~Hz}, 0.5-1.5 \mathrm{~mA}, 30 \mathrm{~min} / \\
\text { time }\end{array}$ & 8 & PWT, BW & NR & $P<0.05$ \\
\hline $\begin{array}{l}\text { Shi et al. } \\
{[42]}\end{array}$ & $\mathrm{EA}(11)$ & $\begin{array}{l}\text { Model }(8) \\
\text { Morphine } \\
\text { tolerance }(11) \\
\text { SEA(9) }\end{array}$ & $\begin{array}{l}\text { ST-36, } \\
\text { BL60 }\end{array}$ & $\begin{array}{c}\mathrm{QD}, 2 / 100 \mathrm{~Hz}, 0.5-1.5 \mathrm{~mA}, \\
30 \mathrm{~min} / \mathrm{time}\end{array}$ & 7 & PWT & NR & $P<0.05$ \\
\hline $\begin{array}{l}\text { Guan } \\
\text { et al. [43] }\end{array}$ & $\begin{array}{c}\mathrm{EA}+\text { herbal } \\
\text { medicine }(20)\end{array}$ & Herbal medicine (20) & $\begin{array}{l}\text { ST36, } \\
\text { GenDuan }\end{array}$ & $\mathrm{QD}, 1-2 \mathrm{~mA}, 30 \mathrm{~min} /$ time & 20 & BW, PWL & NR & $P<0.05$ \\
\hline
\end{tabular}

$\mathrm{EA}=$ electroacupuncture; $\mathrm{PWT}=$ paw withdrawal threshold; $\mathrm{PWL}=$ paw withdrawal latency; $\mathrm{BW}=$ body weight; $\mathrm{PWPT}=$ paw withdrawal pressure threshold; GFAP = glial fibrillary acidic protein; IL- $1 \beta=$ interleukin $-1 \beta$; NR = no report.

the methodological quality of the animal experiments (Table 4). We found that 6 studies describe detailed sequence generation, 15 studies just mentioned randomness but did not provide specific random methods, and 3 studies did even not mention randomization. All the include studies provided baseline characteristics. No included studies mentioned allocation concealment. 21 studies mentioned random housing of the animals. None of the included studies described caregivers and/ or investigators blinded from knowledge of which intervention each animal received during the experiment. None of the studies assess outcome randomly. Four studies described that the outcome assessor was blinded. Eight studies were free of incomplete outcome data and 10 studies were influenced by incomplete outcome data mainly due to model failure; the pity is that they did not inform if the missing outcome data were imputed by appropriate methods. All the included studies were free of selective outcome reporting. Five studies were free of other sources of bias, 13 studies were unclear about their influence by other sources of bias, and 6 studies were influenced by other sources of bias significantly.

\subsection{Results of Meta-Analysis}

\subsubsection{Acupuncture's Effect on PWT}

(1) Acupuncture versus Sham/Placebo Acupuncture or Wait List Group. For a total of six RCTs based on the PWT to evaluate effect (Figure 2(a)), the pooled results showed that EA was prior to sham/placebo or wait list group, with mean difference of 1.50 (95\% CI, 0.35 to $2.64 ; P<0.00001$; $\left.I^{2}=92 \%\right)$.

(2) Acupuncture versus Medicine. Meta-analysis of six RCTs suggested PWT was significantly improved in control groups versus acupuncture groups (Figure 2(b)), with a mean difference of -3.51 ( $95 \% \mathrm{CI},-5.55$ to -1.46 ; $P<0.00001$; $\left.I^{2}=93 \%\right)$. 
TABLE 3: STRICTA 2010 checklist for the included studies.

\begin{tabular}{|c|c|c|c|c|c|c|c|c|c|c|c|c|c|c|c|c|c|}
\hline \multirow{2}{*}{ Study ID } & \multicolumn{3}{|c|}{1} & \multicolumn{7}{|c|}{2} & \multicolumn{2}{|c|}{3} & \multicolumn{2}{|c|}{4} & \multirow{2}{*}{5} & \multicolumn{2}{|c|}{6} \\
\hline & $a$ & $b$ & $c$ & $a$ & $b$ & $c$ & $d$ & $e$ & $f$ & $g$ & $a$ & $b$ & $a$ & $b$ & & $a$ & $b$ \\
\hline Zhang et al. [21] & $\mathrm{Y}$ & $\mathrm{Y}$ & $\mathrm{Y}$ & $\mathrm{Y}$ & $\mathrm{Y}$ & $\mathrm{Y}$ & $\mathrm{Y}$ & $\mathrm{Y}$ & $\mathrm{Y}$ & $\mathrm{U}$ & $\mathrm{Y}$ & $\mathrm{Y}$ & $\mathrm{Y}$ & $\mathrm{N}$ & $\mathrm{N}$ & $\mathrm{N}$ & $\mathrm{Y}$ \\
\hline Qiliang [20] & $\mathrm{Y}$ & $\mathrm{Y}$ & $\mathrm{Y}$ & $\mathrm{Y}$ & $\mathrm{Y}$ & $\mathrm{Y}$ & $\mathrm{Y}$ & $\mathrm{Y}$ & $\mathrm{Y}$ & $\mathrm{N}$ & $\mathrm{Y}$ & $\mathrm{Y}$ & $\mathrm{Y}$ & $\mathrm{N}$ & $\mathrm{N}$ & $\mathrm{N}$ & $\mathrm{Y}$ \\
\hline Zhang et al. [23] & $\mathrm{Y}$ & $\mathrm{Y}$ & $\mathrm{Y}$ & $\mathrm{Y}$ & $\mathrm{Y}$ & $\mathrm{Y}$ & $\mathrm{Y}$ & $\mathrm{Y}$ & $\mathrm{Y}$ & $\mathrm{U}$ & $\mathrm{Y}$ & $\mathrm{Y}$ & $\mathrm{Y}$ & $\mathrm{N}$ & $\mathrm{N}$ & $\mathrm{N}$ & $\mathrm{Y}$ \\
\hline Qiliang et al. [22] & $\mathrm{Y}$ & $\mathrm{Y}$ & $\mathrm{Y}$ & $\mathrm{Y}$ & $\mathrm{Y}$ & $\mathrm{Y}$ & $\mathrm{Y}$ & $\mathrm{Y}$ & $\mathrm{Y}$ & $\mathrm{N}$ & $\mathrm{Y}$ & $\mathrm{Y}$ & $\mathrm{Y}$ & $\mathrm{N}$ & $\mathrm{N}$ & $\mathrm{N}$ & $\mathrm{Y}$ \\
\hline $\operatorname{Li}[24]$ & $\mathrm{Y}$ & $\mathrm{Y}$ & $\mathrm{Y}$ & $\mathrm{Y}$ & $\mathrm{Y}$ & $\mathrm{N}$ & $\mathrm{Y}$ & $\mathrm{Y}$ & $\mathrm{Y}$ & $\mathrm{N}$ & $\mathrm{Y}$ & $\mathrm{Y}$ & $\mathrm{Y}$ & $\mathrm{N}$ & $\mathrm{N}$ & $\mathrm{N}$ & $\mathrm{Y}$ \\
\hline Smeester et al. [25] & $\mathrm{Y}$ & $\mathrm{Y}$ & $\mathrm{Y}$ & $\mathrm{Y}$ & $\mathrm{Y}$ & $\mathrm{Y}$ & $\mathrm{N}$ & $\mathrm{Y}$ & $\mathrm{Y}$ & $\mathrm{U}$ & $\mathrm{Y}$ & $\mathrm{Y}$ & $\mathrm{Y}$ & $\mathrm{N}$ & $\mathrm{N}$ & $\mathrm{N}$ & $\mathrm{Y}$ \\
\hline Kuai et al. [26] & $\mathrm{Y}$ & $\mathrm{Y}$ & $\mathrm{Y}$ & $\mathrm{Y}$ & $\mathrm{Y}$ & $\mathrm{Y}$ & $\mathrm{N}$ & $\mathrm{Y}$ & $\mathrm{Y}$ & $\mathrm{U}$ & $\mathrm{Y}$ & $\mathrm{Y}$ & $\mathrm{Y}$ & $\mathrm{N}$ & $\mathrm{N}$ & $\mathrm{N}$ & $\mathrm{Y}$ \\
\hline Zhao et al. [28] & $\mathrm{Y}$ & $\mathrm{U}$ & $\mathrm{Y}$ & $\mathrm{Y}$ & $\mathrm{Y}$ & $\mathrm{Y}$ & $\mathrm{N}$ & $\mathrm{Y}$ & $\mathrm{Y}$ & $\mathrm{U}$ & $\mathrm{Y}$ & $\mathrm{Y}$ & $\mathrm{Y}$ & $\mathrm{N}$ & $\mathrm{N}$ & $\mathrm{N}$ & $\mathrm{Y}$ \\
\hline Si-ma et al. [27] & $\mathrm{Y}$ & $\mathrm{Y}$ & $\mathrm{Y}$ & $\mathrm{U}$ & $\mathrm{U}$ & $\mathrm{N}$ & $\mathrm{N}$ & $\mathrm{Y}$ & $\mathrm{Y}$ & $\mathrm{N}$ & $\mathrm{Y}$ & $\mathrm{Y}$ & $\mathrm{Y}$ & $\mathrm{N}$ & $\mathrm{N}$ & $\mathrm{N}$ & $\mathrm{Y}$ \\
\hline Zhao et al. [29] & $\mathrm{Y}$ & $\mathrm{Y}$ & $\mathrm{Y}$ & $\mathrm{Y}$ & $\mathrm{Y}$ & $\mathrm{Y}$ & $\mathrm{N}$ & $\mathrm{Y}$ & $\mathrm{Y}$ & $\mathrm{N}$ & $\mathrm{Y}$ & $\mathrm{Y}$ & $\mathrm{Y}$ & $\mathrm{N}$ & $\mathrm{N}$ & $\mathrm{N}$ & $\mathrm{Y}$ \\
\hline Du et al. [30] & $\mathrm{Y}$ & $\mathrm{Y}$ & $\mathrm{Y}$ & $\mathrm{Y}$ & $\mathrm{Y}$ & $\mathrm{N}$ & $\mathrm{N}$ & $\mathrm{Y}$ & $\mathrm{Y}$ & $\mathrm{N}$ & $\mathrm{Y}$ & $\mathrm{Y}$ & $\mathrm{Y}$ & $\mathrm{N}$ & $\mathrm{N}$ & $\mathrm{N}$ & $\mathrm{Y}$ \\
\hline Du et al. [31] & $\mathrm{Y}$ & $\mathrm{Y}$ & $\mathrm{Y}$ & $\mathrm{Y}$ & $\mathrm{Y}$ & $\mathrm{N}$ & $\mathrm{N}$ & $\mathrm{Y}$ & $\mathrm{Y}$ & $\mathrm{N}$ & $\mathrm{Y}$ & $\mathrm{Y}$ & $\mathrm{Y}$ & $\mathrm{N}$ & $\mathrm{N}$ & $\mathrm{N}$ & $\mathrm{Y}$ \\
\hline Lu et al. [32] & $\mathrm{Y}$ & $\mathrm{Y}$ & $\mathrm{Y}$ & $\mathrm{Y}$ & $\mathrm{Y}$ & $\mathrm{Y}$ & $\mathrm{N}$ & $\mathrm{Y}$ & $\mathrm{Y}$ & $\mathrm{U}$ & $\mathrm{Y}$ & $\mathrm{Y}$ & $\mathrm{Y}$ & $\mathrm{N}$ & $\mathrm{N}$ & $\mathrm{N}$ & $\mathrm{Y}$ \\
\hline Lu et al. [33] & $\mathrm{Y}$ & $\mathrm{Y}$ & $\mathrm{Y}$ & $\mathrm{U}$ & $\mathrm{U}$ & $\mathrm{N}$ & $\mathrm{N}$ & $\mathrm{Y}$ & $\mathrm{Y}$ & $\mathrm{N}$ & $\mathrm{Y}$ & $\mathrm{Y}$ & $\mathrm{Y}$ & $\mathrm{N}$ & $\mathrm{N}$ & $\mathrm{N}$ & $\mathrm{Y}$ \\
\hline Sima et al. [34] & $\mathrm{Y}$ & $\mathrm{Y}$ & $\mathrm{Y}$ & $\mathrm{Y}$ & $\mathrm{Y}$ & $\mathrm{Y}$ & $\mathrm{N}$ & $\mathrm{Y}$ & $\mathrm{Y}$ & $\mathrm{U}$ & $\mathrm{Y}$ & $\mathrm{Y}$ & $\mathrm{Y}$ & $\mathrm{N}$ & $\mathrm{N}$ & $\mathrm{N}$ & $\mathrm{Y}$ \\
\hline Fu et al. [35] & $\mathrm{Y}$ & $\mathrm{Y}$ & $\mathrm{Y}$ & $\mathrm{Y}$ & $\mathrm{Y}$ & $\mathrm{N}$ & $\mathrm{N}$ & $\mathrm{Y}$ & $\mathrm{Y}$ & $\mathrm{N}$ & $\mathrm{Y}$ & $\mathrm{Y}$ & $\mathrm{Y}$ & $\mathrm{N}$ & $\mathrm{N}$ & $\mathrm{N}$ & $\mathrm{Y}$ \\
\hline Fu et al. [36] & $\mathrm{Y}$ & $\mathrm{Y}$ & $\mathrm{Y}$ & $\mathrm{Y}$ & $\mathrm{Y}$ & $\mathrm{N}$ & $\mathrm{N}$ & $\mathrm{Y}$ & $\mathrm{Y}$ & $\mathrm{U}$ & $\mathrm{Y}$ & $\mathrm{Y}$ & $\mathrm{Y}$ & $\mathrm{N}$ & $\mathrm{N}$ & $\mathrm{N}$ & $\mathrm{Y}$ \\
\hline Liang et al. [38] & $\mathrm{Y}$ & $\mathrm{Y}$ & $\mathrm{Y}$ & $\mathrm{Y}$ & $\mathrm{Y}$ & $\mathrm{Y}$ & $\mathrm{N}$ & $\mathrm{Y}$ & $\mathrm{Y}$ & $\mathrm{Y}$ & $\mathrm{Y}$ & $\mathrm{Y}$ & $\mathrm{Y}$ & $\mathrm{N}$ & $\mathrm{N}$ & $\mathrm{N}$ & $\mathrm{Y}$ \\
\hline Liang et al. [39] & $\mathrm{Y}$ & $\mathrm{Y}$ & $\mathrm{Y}$ & $\mathrm{Y}$ & $\mathrm{Y}$ & $\mathrm{N}$ & $\mathrm{N}$ & $\mathrm{Y}$ & $\mathrm{Y}$ & $\mathrm{U}$ & $\mathrm{Y}$ & $\mathrm{Y}$ & $\mathrm{Y}$ & $\mathrm{N}$ & $\mathrm{N}$ & $\mathrm{N}$ & $\mathrm{Y}$ \\
\hline Guan et al. [37] & $\mathrm{Y}$ & $\mathrm{Y}$ & $\mathrm{Y}$ & $\mathrm{Y}$ & $\mathrm{Y}$ & $\mathrm{N}$ & $\mathrm{Y}$ & $\mathrm{Y}$ & $\mathrm{Y}$ & $\mathrm{N}$ & $\mathrm{Y}$ & $\mathrm{Y}$ & $\mathrm{Y}$ & $\mathrm{N}$ & $\mathrm{N}$ & $\mathrm{N}$ & $\mathrm{Y}$ \\
\hline Qin et al. [40] & $\mathrm{Y}$ & $\mathrm{Y}$ & $\mathrm{Y}$ & $\mathrm{Y}$ & $\mathrm{Y}$ & $\mathrm{Y}$ & $\mathrm{Y}$ & $\mathrm{Y}$ & $\mathrm{Y}$ & $\mathrm{N}$ & $\mathrm{Y}$ & $\mathrm{Y}$ & $\mathrm{Y}$ & $\mathrm{N}$ & $\mathrm{N}$ & $\mathrm{N}$ & $\mathrm{Y}$ \\
\hline Cai et al. [41] & $\mathrm{Y}$ & $\mathrm{Y}$ & $\mathrm{Y}$ & $\mathrm{Y}$ & $\mathrm{Y}$ & $\mathrm{Y}$ & $\mathrm{Y}$ & $\mathrm{Y}$ & $\mathrm{Y}$ & $\mathrm{U}$ & $\mathrm{Y}$ & $\mathrm{Y}$ & $\mathrm{Y}$ & $\mathrm{N}$ & $\mathrm{N}$ & $\mathrm{N}$ & $\mathrm{Y}$ \\
\hline Shi et al. [42] & $\mathrm{Y}$ & $\mathrm{Y}$ & $\mathrm{Y}$ & $\mathrm{Y}$ & $\mathrm{Y}$ & $\mathrm{N}$ & $\mathrm{N}$ & $\mathrm{Y}$ & $\mathrm{Y}$ & $\mathrm{U}$ & $\mathrm{Y}$ & $\mathrm{Y}$ & $\mathrm{Y}$ & $\mathrm{N}$ & $\mathrm{N}$ & $\mathrm{N}$ & $\mathrm{Y}$ \\
\hline Guan et al. [43] & $\mathrm{Y}$ & $\mathrm{Y}$ & $\mathrm{Y}$ & $\mathrm{Y}$ & $\mathrm{Y}$ & $\mathrm{N}$ & $\mathrm{N}$ & $\mathrm{Y}$ & $\mathrm{Y}$ & $\mathrm{N}$ & $\mathrm{Y}$ & $\mathrm{Y}$ & $\mathrm{Y}$ & $\mathrm{N}$ & $\mathrm{N}$ & $\mathrm{N}$ & $\mathrm{Y}$ \\
\hline
\end{tabular}

$\mathrm{Y}=$ yes; $\mathrm{N}=$ no; $\mathrm{U}=$ unclear.

TABLE 4: SYRCLE risk of bias tool for included studies.

\begin{tabular}{|c|c|c|c|c|c|c|c|c|c|c|c|}
\hline D & 1 & 2 & 3 & 4 & 5 & 6 & 7 & 8 & 9 & 10 & \\
\hline & & Y & $\mathrm{N}$ & $\mathrm{Y}$ & $\mathrm{U}$ & $\mathrm{U}$ & 1 & $\mathrm{U}$ & $\mathrm{Y}$ & & \\
\hline & & 1 & 0 & 1 & & & & & & & \\
\hline & & $\mathrm{Y}$ & $\mathrm{U}$ & $\mathrm{Y}$ & $\mathrm{U}$ & $\mathrm{U}$ & $\mathrm{Y}$ & & & $\mathrm{U}$ & \\
\hline & & $\mathrm{Y}$ & $\mathrm{U}$ & $\mathrm{Y}$ & $\mathrm{U}$ & $\mathrm{N}$ & $\mathrm{U}$ & $\mathrm{Y}$ & & $\mathrm{Y}$ & \\
\hline & & $\mathrm{Y}$ & $\mathrm{U}$ & $\mathrm{Y}$ & $\mathrm{U}$ & $\mathrm{N}$ & $\mathrm{U}$ & $\mathrm{Y}$ & & $\mathrm{U}$ & \\
\hline & $\mathrm{N}$ & $\mathrm{Y}$ & $\mathrm{N}$ & $\mathrm{Y}$ & $\mathrm{U}$ & $\mathrm{N}$ & $\mathrm{Y}$ & $\mathrm{N}$ & & $\mathrm{N}$ & \\
\hline & $\mathrm{V}$ & $\mathrm{Y}$ & $\mathrm{U}$ & $\mathrm{Y}$ & $\mathrm{U}$ & $\mathrm{N}$ & $\mathrm{U}$ & $\mathrm{N}$ & & $\mathrm{N}$ & \\
\hline & & $\mathrm{Y}$ & $\mathrm{U}$ & $\mathrm{Y}$ & $\mathrm{U}$ & $\mathrm{U}$ & $\mathrm{U}$ & $\mathrm{U}$ & $\mathrm{Y}$ & $\mathrm{U}$ & \\
\hline & $\mathrm{Y}$ & $\mathrm{Y}$ & $\mathrm{U}$ & $\mathrm{U}$ & $\mathrm{U}$ & $\mathrm{U}$ & $\mathrm{U}$ & $\mathrm{U}$ & $\mathrm{Y}$ & $\mathrm{U}$ & \\
\hline & & $\mathrm{Y}$ & $\mathrm{U}$ & $\mathrm{Y}$ & $\mathrm{U}$ & $\mathrm{N}$ & $\mathrm{U}$ & $\mathrm{Y}$ & & $\mathrm{U}$ & \\
\hline & & $\mathrm{Y}$ & $\mathrm{U}$ & $\mathrm{Y}$ & $\mathrm{U}$ & $\mathrm{N}$ & $\mathrm{U}$ & $\mathrm{Y}$ & & 2 & \\
\hline & & $\mathrm{Y}$ & $\mathrm{U}$ & $\mathrm{Y}$ & $\mathrm{U}$ & $\mathrm{N}$ & $\mathrm{Y}$ & $\mathrm{N}$ & $\mathrm{Y}$ & $\mathrm{N}$ & \\
\hline & & $\mathrm{Y}$ & $\mathrm{U}$ & $\mathrm{Y}$ & $\mathrm{U}$ & $\mathrm{N}$ & $\mathrm{U}$ & $\mathrm{Y}$ & $\mathrm{Y}$ & $\mathrm{U}$ & \\
\hline & & $\mathrm{Y}$ & $\mathrm{U}$ & $\mathrm{U}$ & $\mathrm{U}$ & $\mathrm{U}$ & $\mathrm{U}$ & $\mathrm{U}$ & $\mathrm{Y}$ & $\mathrm{U}$ & \\
\hline & & $\mathrm{Y}$ & $\mathrm{U}$ & $\mathrm{Y}$ & $\mathrm{U}$ & $\mathrm{U}$ & $\mathrm{U}$ & $\mathrm{U}$ & & $\mathrm{Y}$ & \\
\hline & & $\mathrm{Y}$ & $\mathrm{U}$ & $\mathrm{Y}$ & $\mathrm{U}$ & $\mathrm{U}$ & $\mathrm{U}$ & $\mathrm{U}$ & $\mathrm{Y}$ & $\mathrm{Y}$ & \\
\hline & & $\mathrm{Y}$ & $\mathrm{U}$ & $\mathrm{Y}$ & $\mathrm{U}$ & $\mathrm{N}$ & $\mathrm{U}$ & $\mathrm{U}$ & $\mathrm{Y}$ & $\mathrm{U}$ & \\
\hline & & $\mathrm{Y}$ & $\mathrm{U}$ & $\mathrm{Y}$ & $\mathrm{U}$ & $\mathrm{U}$ & $\mathrm{U}$ & $\mathrm{U}$ & $\mathrm{Y}$ & $\mathrm{U}$ & \\
\hline & & $\mathrm{Y}$ & $\mathrm{U}$ & $\mathrm{Y}$ & $\mathrm{U}$ & $\mathrm{N}$ & $\mathrm{U}$ & $\mathrm{U}$ & $\mathrm{Y}$ & $\mathrm{N}$ & \\
\hline & & $\mathrm{Y}$ & $\mathrm{U}$ & $\mathrm{U}$ & $\mathrm{U}$ & $\mathrm{N}$ & $\mathrm{U}$ & $\mathrm{Y}$ & $\mathrm{Y}$ & $\mathrm{U}$ & $1 \mathrm{~N}$ \\
\hline & & $\mathrm{Y}$ & $\mathrm{N}$ & $\mathrm{Y}$ & $\mathrm{U}$ & $\mathrm{U}$ & $\mathrm{U}$ & $\mathrm{U}$ & $\mathrm{Y}$ & $\mathrm{U}$ & \\
\hline & & $\mathrm{Y}$ & $\mathrm{U}$ & $\mathrm{Y}$ & $\mathrm{U}$ & $\mathrm{N}$ & $\mathrm{U}$ & $\mathrm{N}$ & $\mathrm{Y}$ & $\mathrm{N}$ & \\
\hline & & $\mathrm{Y}$ & $\mathrm{U}$ & Y & $\mathrm{U}$ & $\mathrm{N}$ & $\mathrm{U}$ & $\mathrm{N}$ & Y & $\mathrm{N}$ & \\
\hline thant & & & & & & & & & & & $T^{2}$ \\
\hline
\end{tabular}

$\mathrm{Y}=$ yes; $\mathrm{N}=$ no; $\mathrm{U}=$ unclear.

(3) Acupuncture Combined with Medicine versus Single Medicine. Meta-analysis of five RCTs suggested a significant improvement in PWT in acupuncture combined with medicine groups versus single medicine group (Figure 2(c)), with a mean difference of 1.25 (95\% CI, 0.56 to $1.95 ; P=0.03$; $\left.I^{2}=62 \%\right)$.

\subsubsection{Acupuncture's Effect on PWL}

(1) Acupuncture versus Sham/Placebo Acupuncture or Model. Meta-analysis of five RCTs suggested PWL was improved significantly by acupuncture compared with sham/placebo acupuncture or wait list group (Figure 3(a)), with a mean difference of $1.52(95 \%, 0.61$ to $2.44 ; P=0.005$; $\left.I^{2}=73 \%\right)$.

(2) Acupuncture versus Medicine. Meta-analysis of four RCTs showed that acupuncture had no less effect than medicine in terms of improving PWL (Figure 3(b)), with a mean difference of $-0.04\left(95 \%,-0.47\right.$ to $\left.0.39 ; P=0.76 ; I^{2}=0 \%\right)$.

(3) Acupuncture Combined with Medicine versus Single Medicine. Meta-analysis of five RCTs suggested acupuncture combined with medicine was more effective than single medicine (Figure 3(c)) on PWL, with a mean difference of 1.26 (95\% CI, 0.05 to $2.47 ; P<0.00001 ; I^{2}=88 \%$ ).

3.4.3. Subgroup Analysis according to Animal Species Based on PWL. To investigate the source of heterogeneity, we developed subgroup analysis according to animal species based on PWL (Figure 4). The heterogeneity decreased after 


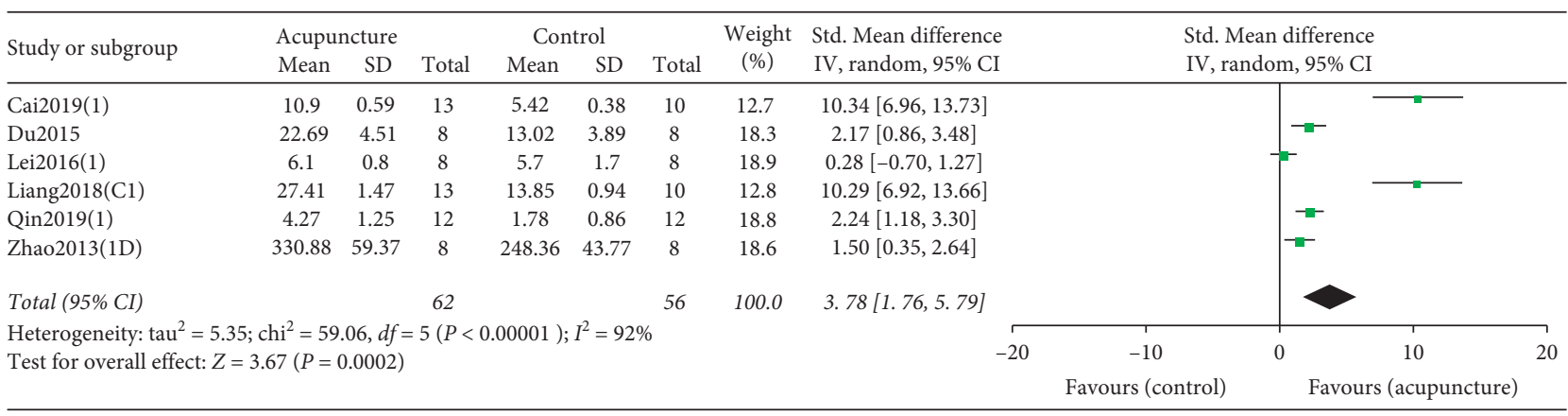

(a)

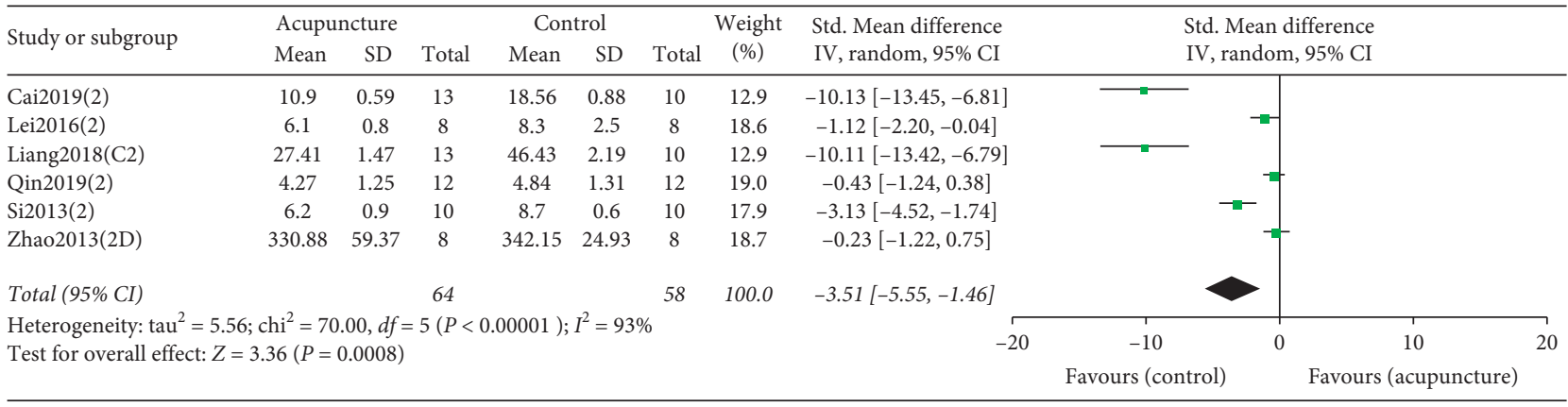

(b)

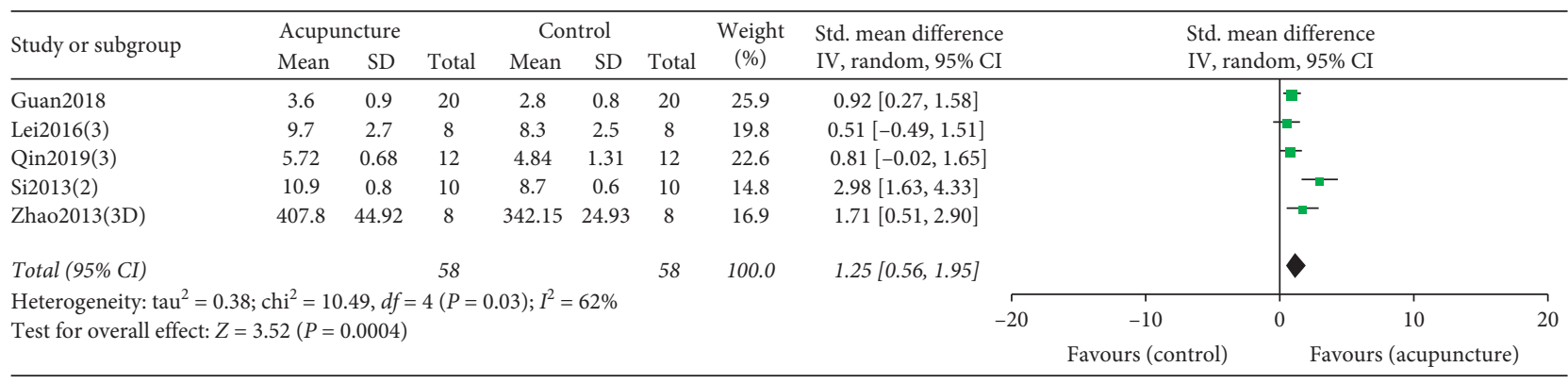

(c)

FIGURE 2: (a). Forest plot comparing paw withdrawal threshold between acupuncture and model. (b). Forest plot comparing paw withdrawal threshold between acupuncture and medicine. (c). Forest plot comparing paw withdrawal threshold between single medicine and medicine combined with acupuncture. The black diamond represents the point estimate and $95 \%$ confidence interval. The green block at the point estimate of intervention effect with a horizontal line extending either side of the block represents a study. The area of the block indicates the weight assigned to that study in the meta-analysis while the horizontal black line depicts the $95 \%$ confidence interval. Statistical method is inverse variance. Random effects model was employed as analysis model. The effect measure was standard mean difference.

subgroup analysis, which indicates that animal species maybe one of the sources of heterogeneity.

3.4.4. Acupuncture's Effect on Body Weight. Meta-analysis of six RCTs suggested acupuncture (or acupuncture combined with medicine) was prior to control group (including single medicine, sham/placebo acupuncture, and wait list group) in improving model animals' body weight (Figure 5(a)), with a mean difference of 0.83 ( $95 \% \mathrm{CI}, 0.14$ to $\left.1.51 ; P=0.006 ; I^{2}=69 \%\right)$.

3.4.5. Acupuncture's Effect on GFAP. Meta-analysis of eight RCTs showed acupuncture (or acupuncture combined with medicine) could decrease model animals' spinal cord GFAP significantly compared to control group (including single medicine, sham/placebo acupuncture, and wait list group) (Figure 5(b)), with a mean difference of -1.36 (95\% CI, -2.27 to $\left.-0.45 ; P<0.00001 ; I^{2}=79 \%\right)$.

3.4.6. Acupuncture's Effect on IL-1 $\beta$. Meta-analysis of four RCTs suggested acupuncture (or acupuncture combined with medicine) could decrease model animals' spinal cord IL- $1 \beta$ significantly compared to control group (including single medicine, sham/placebo acupuncture, and wait list group) (Figure 5(c)), with a mean difference of -1.55 (95\% CI, -2.52 to $-0.59 ; P=0.003 ; I^{2}=78 \%$ ). 


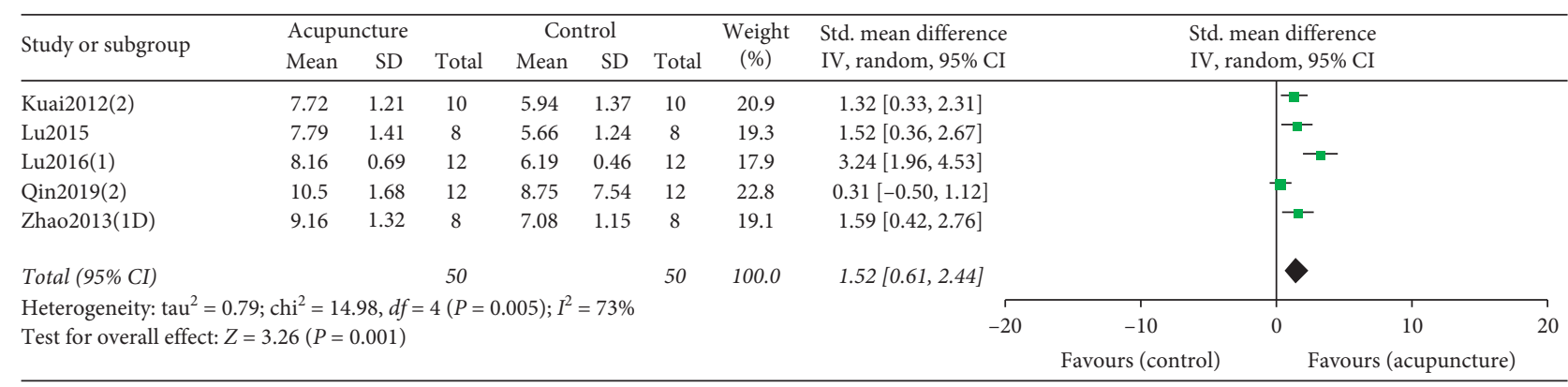

(a)

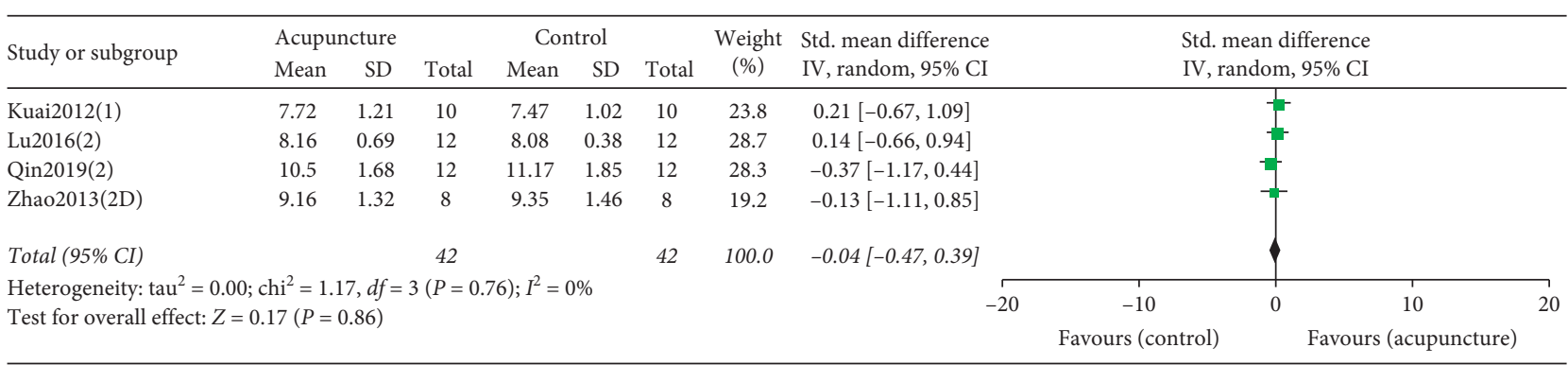

(b)

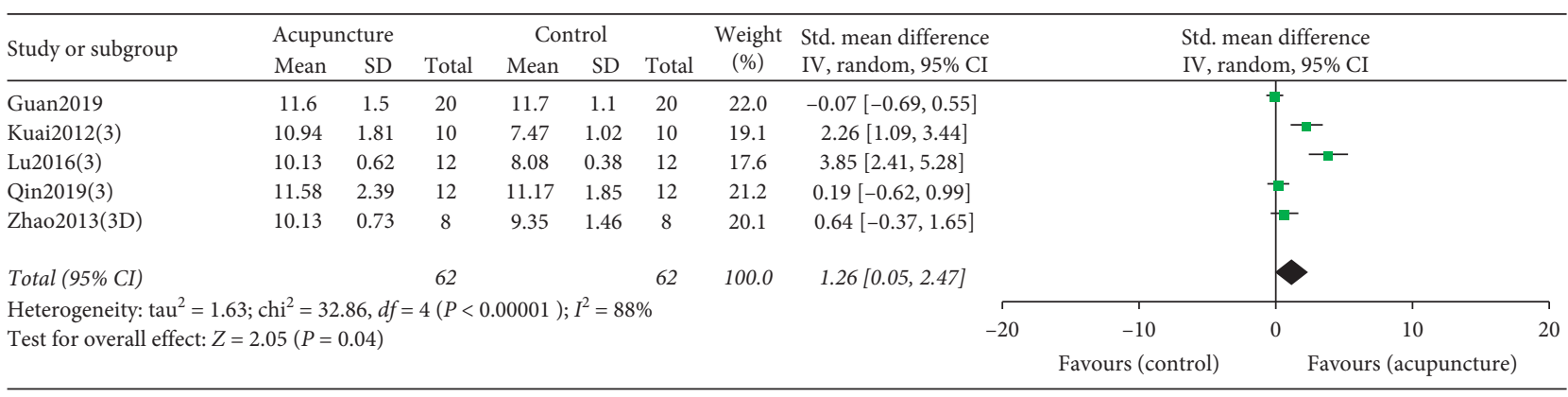

(c)

FIGURE 3: (a). Forest plot comparing paw withdrawal latency between acupuncture and model. (b). Forest plot comparing paw withdrawal latency between acupuncture and medicine. (c). Forest plot comparing paw withdrawal latency between single medicine and medicine combined with acupuncture. The black diamond represents the point estimate and $95 \%$ confidence interval. The green block at the point estimate of intervention effect with a horizontal line extending either side of the block represents a study. The area of the block indicates the weight assigned to that study in the meta-analysis while the horizontal black line depicts the $95 \%$ confidence interval. Statistical method is inverse variance. Random effects model was employed as analysis model. The effect measure was standard mean difference.

3.5. Studies that Cannot Be Pooled by Meta-Analysis. Of the 12 studies included in the qualitative synthesis [Table 5], 3 explored the EA effects on morphine tolerance [35, 36, 42]. These researches showed that compared to bone cancer pain (BCP) group, morphine tolerance group, and sham EA group, EA can improve mechanical pain threshold in rats with bone cancer pain significantly. What is more, the analgesia effect of EA at different time point on morphine tolerance in rats with BCP showed that early intervention of EA stimulation before the formation of morphine tolerance could delay it in rats with BCP [35]; EA stimulation after the formation of morphine tolerance could partially reverse it in rats with BCP. Of the eight studies $[21,23,24,26,30,35,36,42]$, comparing the effect of EA with sham EA on CIBP rats' thermal pain threshold or mechanical pain threshold, seven studies $[21,23-25,30,35,36]$ showed that EA is prior to SEA in relieving CIBP. One study [24] showed that neither single nor multiple EA treatment displayed significant analgesia effect on CIBP. Two studies [25, 30] explored how the scheduling regimens and parameters influence the effect of EA on CIBP. One study [25] demonstrated acupuncture at any time showed immediate antinociceptive effect, but acupuncture twice weekly beginning on implantation day 3 or prophylactically three times prior to implantation produced the most robust and longest lasting antinociceptive effect. What is more, gender difference was found in analgesic effect of acupuncture. Another study [30] indicated that EA treatment once had a good analgesic effect on CIBP; the analgesic effect is not related to EA frequency. For EA treatment for a long time, the analgesic effect of EA treatment once every other day is better than EA treatment once a day. Two studies $[20,22]$ indicated that EA alone has no effect on CIBP, but EA can make small dose of Celebrex, 


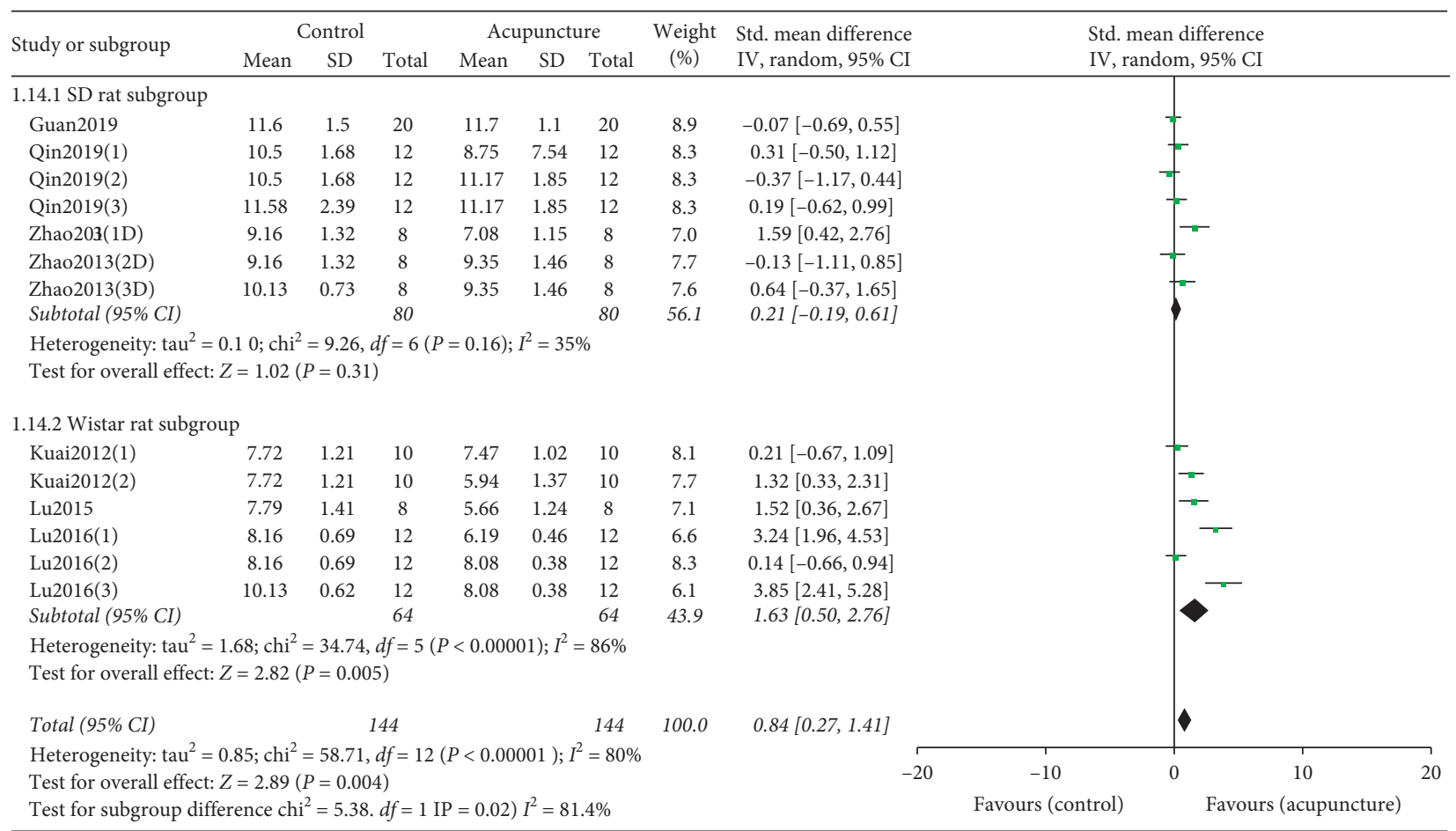

FIGURE 4: Forest plot of subgroup analysis according to animal species based on PWL. SD rat subgroup: forest plot of SD rat subgroup analyzation comparing PWL between acupuncture and control group. Wistar rat subgroup: forest plot of Wistar rat subgroup analyzation comparing PWL between acupuncture and control group. The black diamond represents the point estimate and $95 \%$ confidence interval. The green block at the point estimate of intervention effect with a horizontal line extending either side of the block represents a study. The area of the block indicates the weight assigned to that study in the meta-analysis while the horizontal black line depicts the $95 \%$ confidence interval. Statistical method is inverse variance. Random effects model was employed as analysis model. The effect measure was standard mean difference.

which appears useless in relieving CIBP, exhibiting prominent analgesic effect. One study [38] demonstrated that both EA and morphine were effective on CIBP, while EA's analgesic effect was weaker compared with morphine.

3.6. Publication Bias. To address publication bias, we performed funnel plots (Figure 6(a)) for the studies included in quantitative synthesis. No asymmetric pattern was seen. Both Egger $(P=0.016)$ and Begg's test $(P=0.512)$ showed that there was no publication bias (Figure 6(b)).

STRICTA 2010 checklist showed that the following items were reported well: style of acupuncture, reasoning for treatment provided, extent to which treatment was varied, number of needle insertions per subject per session, names of points used, needle stimulation, needle retention time, number of treatment sessions, frequency and duration of treatment sessions, details of other interventions administered to the acupuncture group, and precise description of the control or comparator. At least 22 of the 24 trials reported detailed information of the above items. 11 trials reported the depth of insertion, 8 trials described response sought, and 1 trial demonstrated needle type. None of the included studies prescribed the following items: setting and context of treatment; description of participating acupuncturists; and rationale for the control or comparator in the context of the research question. So future studies should provide more information about the setting and context of treatment, acupuncturists' background, and rationale for the control or comparator in the context of the research question.

As SYRCLE checklist, the following items were reported well: baseline characteristics, selective report, and random housing animals. None of the following items were reported in any of the included studies: allocation concealment, caregivers and/or investigators' blinding, and random outcome assessment. Some items were poorly reported: sequence generation, outcome assessor blinding, incomplete outcome data influence, and other sources of bias.

\section{Discussion}

The object of this systematic review and meta-analysis is to evaluate the effectiveness and safeness of acupuncture in treating cancer-induced bone pain. The meta-analysis found that, compared to model group, EA could increase CIBP animals' PWT and PWL significantly and, compared to medicine treatment, EA has no less effect on PWL. As combined with medicine, EA could enforce single medicine's effect in terms of PWT and PWL. Compared to placebo control group or other treatment, EA increases CIBP animas' body weight. Meanwhile, the mechanical research indicates acupuncture could 


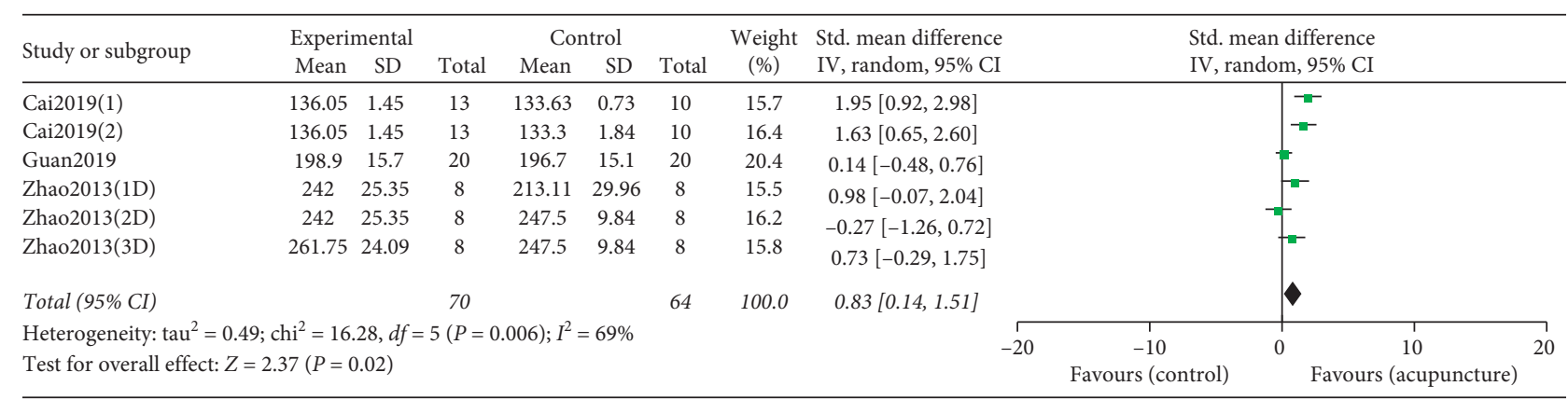

(a)

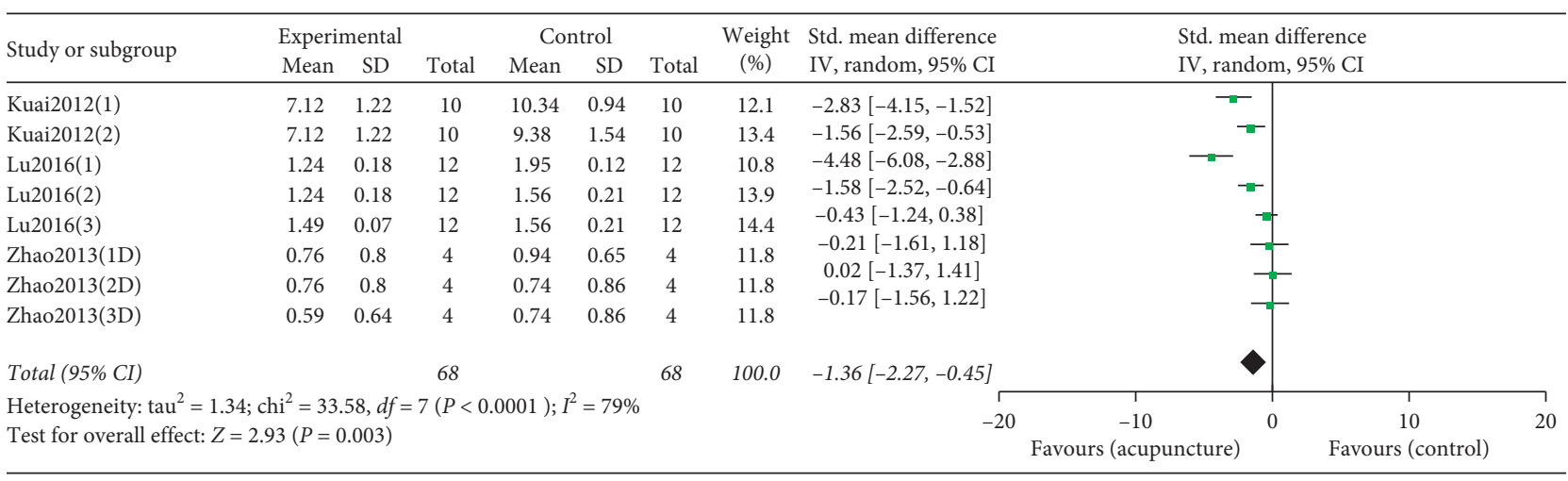

(b)

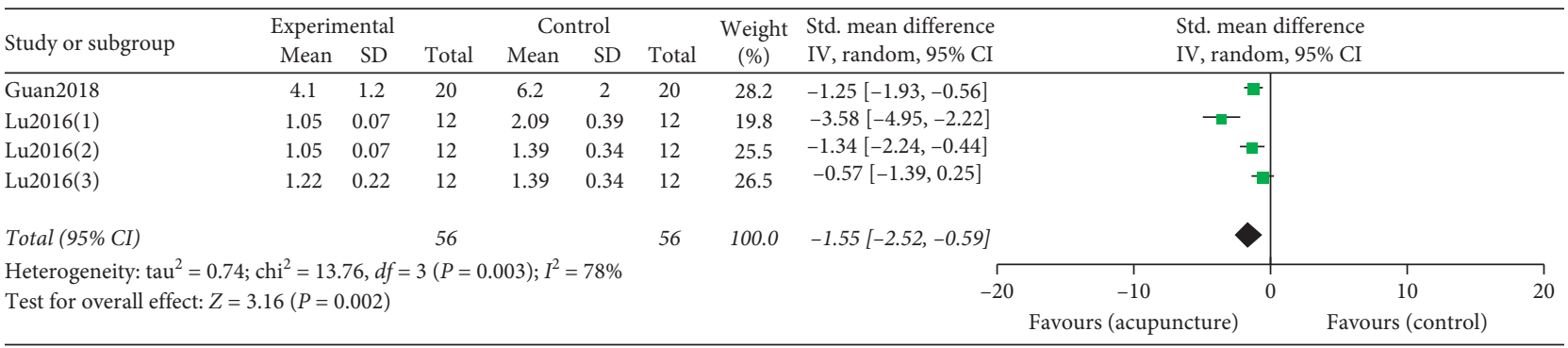

(c)

FIGURE 5: (a). Forest plot comparing body weight between acupuncture and model. (b). Forest plot comparing spinal cord GFAP between acupuncture and medicine. (c). Forest plot comparing spinal cord IL-1 $\beta$ between single medicine and medicine combined with acupuncture. The black diamond represents the point estimate and 95\% confidence interval. The green block at the point estimate of intervention effect with a horizontal line extending either side of the block represents a study. The area of the block indicates the weight assigned to that study in the meta-analysis while the horizontal black line depicts the 95\% confidence interval. Statistical method is inverse variance. Random effects model was employed as analysis model. The effect measure was standard mean difference.

reduce CIBP animals' spinal cord GFAP and IL- $1 \beta$ effectively.

The remaining 12 trials did not pool with metaanalysis due to the fact that no detailed data could be acquired. Three of these researches showed that EA could delay and partially reverse morphine tolerance in rats with BCP. Of the eight studies comparing the effect of EA with sham EA on CIBP rats' PWT and PWL, seven showed that EA is prior to SEA in relieving CIBP. One study showed that neither single nor multiple EA treatment displayed significant analgesia effect on CIBP. One study demonstrated acupuncture at any time showed immediate antinociceptive effect, but the most robust and longest lasting antinociceptive effect is only seen as acupuncture twice weekly beginning on implantation day 3 or prophylactically three times prior to implantation produced. Additionally, sex differences were found. Another study indicated that EA treatment once had a good analgesic effect on CIBP, which was not influenced by EA frequency. As the long time treatment, the analgesic effect of EA once every other day was better than EA treatment once a day. Two studies indicated that rats with CIBP cannot benefit from EA alone, but EA can strengthen small dose Celebrex's analgesic effect. One study demonstrated that both EA and morphine were effective on CIBP, while EA's analgesic effect was weaker compared with morphine.

The major implications for future experimental studies are as follows: firstly, the future animal studies should be 
TABLE 5: Studies that cannot be pooled by meta-analysis.

\begin{tabular}{|c|c|c|c|}
\hline Study ID & EA group & Control group & Potential mechanism \\
\hline Qiliang [20] & $\mathrm{PWT} \longrightarrow$ & $\mathrm{PWT} \uparrow(\mathrm{EA}+$ Celebrex $)$ & $\mathrm{X}$ \\
\hline $\begin{array}{l}\text { Qiliang et al. } \\
\text { [22] }\end{array}$ & $\mathrm{PWT} \longrightarrow$ & $\mathrm{PWT} \uparrow(\mathrm{EA}+$ Celebrex $)$ & $\mathrm{X}$ \\
\hline $\begin{array}{l}\text { Zhang et al. } \\
\text { [21] }\end{array}$ & $\mathrm{PWL} \uparrow$ & $\mathrm{PWL} \longrightarrow(\mathrm{SEA})$ & EA suppresses the IL- $1 \beta$ \\
\hline $\begin{array}{l}\text { Zhang et al. } \\
\text { [23] }\end{array}$ & $\mathrm{PWL} \uparrow \mathrm{PWPT} \uparrow$ & $\mathrm{PWL} \longrightarrow \mathrm{PWPT} \longrightarrow(\mathrm{SEA})$ & EA decreases PPD mRNA \\
\hline $\mathrm{Li}[24]$ & $\mathrm{PWT} \longrightarrow$ & $\mathrm{PWT} \longrightarrow(\mathrm{SEA})$ & $\mathrm{X}$ \\
\hline $\begin{array}{l}\text { Smeester et al. } \\
{[25]}\end{array}$ & $\mathrm{PWT} \uparrow$ & $\mathrm{PWT} \longrightarrow(\mathrm{SEA})$ & EA reduces tumor-associated neutrophils and PGE2 \\
\hline $\begin{array}{l}\text { Zhao et al. } \\
\text { [28] }\end{array}$ & $\mathrm{PWT} \uparrow \mathrm{PWL} \uparrow$ & $\mathrm{PWT} \longrightarrow \mathrm{PWL} \longrightarrow($ Model $)$ & The contents of rats hypothalamic $\beta$-endorphin were restrained \\
\hline Du et al. [30] & $\operatorname{PWT} \uparrow$ & $\mathrm{PWT} \longrightarrow(\mathrm{SEA}$, model $)$ & $\mathrm{X}$ \\
\hline Fu et al. [35] & $\operatorname{PWT} \uparrow$ & $\begin{array}{l}\mathrm{PWT} \longrightarrow(\mathrm{SEA}) \\
\mathrm{PWT} \longrightarrow(\mathrm{MT})\end{array}$ & $\mathrm{X}$ \\
\hline Fu et al. [36] & $\mathrm{PWT} \uparrow$ & $\begin{array}{l}\mathrm{PWT} \longrightarrow(\mathrm{SEA}) \\
\mathrm{PWT} \longrightarrow(\mathrm{MT})\end{array}$ & Improve the MOR positive cells expression in nucleus coeruleus \\
\hline $\begin{array}{l}\text { Liang et al. } \\
{[38]}\end{array}$ & $\mathrm{PWT} \uparrow$ & $\begin{array}{l}\text { PWT } \uparrow \text { (morphine) } \\
\text { (EA }<\text { morphine) }\end{array}$ & $\begin{array}{l}\text { EA increased splenic Con A-induced T cell proliferation and plasma IL-2 } \\
\text { content and increased the percentages of splenic CD3+ CD4+ and CD3+ } \\
\text { CD8+ T cell subsets }\end{array}$ \\
\hline Shi et al. [42] & $\mathrm{PWT} \uparrow$ & $\begin{array}{l}\mathrm{PWT} \longrightarrow(\text { Model }) \\
\mathrm{PWT} \longrightarrow(\mathrm{SEA}) \\
\mathrm{PWT} \longrightarrow(\mathrm{MT})\end{array}$ & $\begin{array}{c}\text { Increase MOR expression and promote endocytosis of MOR in locus } \\
\text { coeruleus region }\end{array}$ \\
\hline
\end{tabular}

PWT = paw withdrawal threshold; $\mathrm{PWL}=$ paw withdrawal latency; $\mathrm{PWPT}=$ paw withdrawal. Pressure threshold; EA= electroacupuncture; $\mathrm{SEA}=$ sham electroacupuncture; $\mathrm{PPD}=$ preprodynorphin; $\mathrm{MT}=$ morphine tolerance; $\mathrm{PGE} 2=$ prostaglandin $E_{2} ; \mathrm{Con} \mathrm{A}=$ concanavalin. $\mathrm{A} ; \mathrm{MOR}=\mu$-opioid receptor; $\uparrow=$ increase; $\longrightarrow=$ no change; $\mathrm{x}=$ no report.

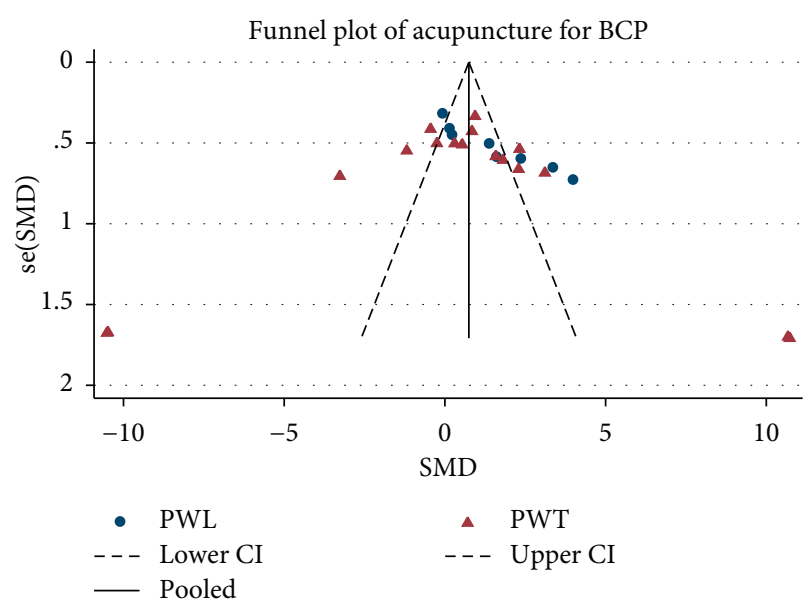

(a)

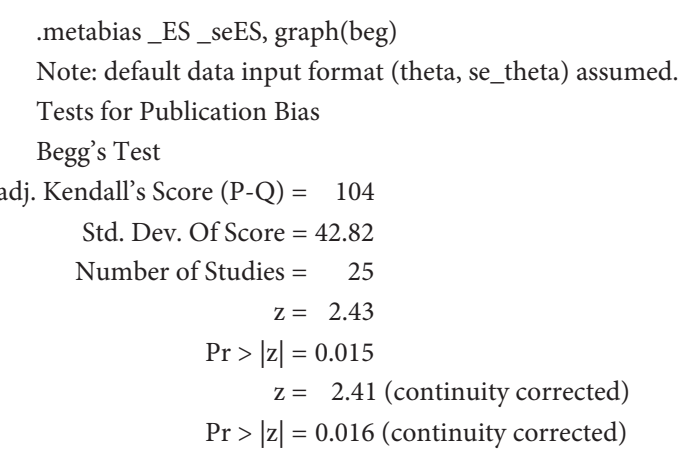

\begin{tabular}{|c|c|c|c|c|c|}
\hline$\overline{\text { Std_Eff }}$ & Coef. & Std. Err. & $\mathrm{t}$ & $\mathrm{P}>|\mathrm{t}|$ & [95\% Conf. Interval] \\
\hline slope & 0.0711634 & 1.083915 & 0.07 & 0.948 & -2.1710852 .313412 \\
\hline bias & 1.348581 & 2.024553 & 0.67 & 0.512 & -2.8395275 .536688 \\
\hline
\end{tabular}

(b)

FIGURE 6: (a). Depiction of publication bias for the paw withdrawal threshold and paw withdrawal latency between acupuncture and control. The red triangle represents the study numbers referring to the paw withdrawal threshold. The blue circle represents the study numbers referring to the paw withdrawal latency. The dashed lines indicated the triangular region with pseudo 95\% confidence interval. (b). Begg's and Egger's test for publication bias. CI: confidence interval; SMD: standard mean difference; PWT: paw withdrawal threshold; PWL: paw withdrawal latency.

designed referring to the SYRCLE to minimize risk of bias and increase the quality of study. Secondly, the future articles should provide the background of acupuncturist, due to the fact that the experience of handlers may influence the efficacy of acupuncture greatly. Thirdly, future studies should increase the sample size to get more reliable conclusion. Lastly, the current evidence of our research indicates that CIBP rats benefit from EA, and maybe clinician can employ EA as a conservative treatment for patients who suffer from caner-induced bone pain.

The key strength of this study is that we have used a meta-analysis drawing on an individual animal data from 
randomized controlled trials of acupuncture for cancerinduced bone pain, which found that EA was superior to both sham and nonacupuncture controls for cancer-induced bone pain condition. However, there were several limitations in this systematic review. Only 5 of the 24 studies were published in English; the remaining studies were published in Chinese. A great majority of the included studies were carried out by Chinese researchers. These elements may induce publication bias. The treatment durations of each study vary greatly. The shortest treatment period was 5 days, while the longest was 20 days. The modeling methods used in the articles were variable. 19 studies used the Walker256 strains to induce neoplasm, 2 studies used the AT-3.1 to induce neoplasm, 1 study used K7M2 to induce neoplasm, and 2 articles used MRMT- 1 to induce neoplasm. The above elements may increase the heterogeneity of the meta-analysis. Only 4 RCTs mentioned outcome assessor blinding, which may induce selective bias. No article described whether the caregivers and/or investigators were blinded from knowledge of which intervention each animal received during the experiment, which may contribute to subjective bias. There was no study reporting the calculation of sample size. Little included studies mentioned the detailed methods of randomization. The above factors greatly reduce the quality of the included studies, which is the key stone of the quality of meta-analysis. So more high quality and larger sample size RCTs are necessary to get a reasonable conclusion.

\section{Conclusion}

As far as we know, this is the first systematic review and meta-analysis to summarize acupuncture for cancer-induced bone pain in animal model. In brief, this metaanalysis and systematic review indicates that, in animal models, electroacupuncture can be used to relieve CIBP, or at least as an assistant treatment. Given the poor quality of included trials, the current existing evidence allows limited conclusions to be reached through comparing acupuncture and medicine, and additional trials are needed to improve the reliability of these findings. Future animal studies should be designed under the guidance of the STRICTA 2010 checklist and SYRCLE risk of bias tool to increase study's quality. Besides, clinical studies are necessary to assess the effect of acupuncture on patients suffering from CIBP.

\section{Disclosure}

The funders have no role in study design, data collection and analysis, decision to publish, or preparation of the manuscript.

\section{Conflicts of Interest}

The authors declare that they have no conflicts of interests.

\section{Authors' Contributions}

Sheng-feng Lu, Mei-ling Yu, and Zhi-gang Lu conceived and designed the experiments; Mei-ling Yu and Jia-jia Qian performed the experiments; and Jia-ying Chen and Yu-wen Zheng performed data extraction. Mei-ling Yu, Shu-Ping Fu, and Sheng-feng $\mathrm{Lu}$ analyzed the data. Mei-ling $\mathrm{Yu}$ and Sheng-feng Lu wrote the paper. sheng-feng Lu, Mei-ling Yu, and Zhi-gang Lu revised the manuscript. All the authors have read and approved the final manuscript.

\section{Acknowledgments}

This work was supported by the National Natural Science Foundation of China (nos. 81774210 and 81574062), "Six Major Talent Summit" of Jiangsu Province (YY-033), Jiangsu Province "333 High-Level Talents Cultivating Project” (2016), "Qing Lan Project” of Jiangsu Province, and a Project Funded by the Nursing Priority Academic Program Development of Jiangsu Higher Education Institutions (2019YSHL059 and 2019YSHL046).

\section{References}

[1] K. L. Pan, W. H. Chan, and Y. Y. Chia, "Initial symptoms and delayed diagnosis of osteosarcoma around the knee joint," Journal of Orthopaedic Surgery, vol. 18, no. 1, pp. 55-57, 2010.

[2] J. L. Ferguson and S. P. Turner, "Bone cancer: diagnosis and treatment principles," American Family Physician, vol. 98, no. 4, pp. 205-213, 2018.

[3] M. Dómine Gómez, N. Díaz Fernández, B. Cantos Sánchez de Ibargüen et al., "Association of performance status and pain in metastatic bone pain management in the Spanish clinical setting," Advances in Therapy, vol. 34, no. 1, pp. 136-147, 2017.

[4] S. Marino and G. D. Roodman, "Multiple myeloma and bone: the fatal interaction," Cold Spring Harbor Perspectives in Medicine, vol. 8, no. 8, 2018.

[5] A. Caraceni, G. Hanks, S. Kaasa et al., "Use of opioid analgesics in the treatment of cancer pain: evidence-based recommendations from the EAPC," The Lancet Oncology, vol. 13, no. 2, pp. e58-e68, 2012.

[6] L. M. Lueptow, A. K. Fakira, and E. N. Bobeck, "The contribution of the descending pain modulatory pathway in opioid tolerance," Front Neurosci, vol. 12, p. 886, 2018.

[7] D. F. Haugen, M. J. Hjermstad, N. Hagen, A. Caraceni, and S. Kaasa, "Assessment and classification of cancer breakthrough pain: a systematic literature review," Pain, vol. 149, no. 3, pp. 476-482, 2010.

[8] S. Mercadante, "Breakthrough pain in cancer patients," Current Opinion in Anaesthesiology, vol. 28, no. 5, pp. 559$564,2015$.

[9] L. Menéndez, A. Lastra, A. Hidalgo, Á. Meana, E. García, and A. Baamonde, "Peripheral opioids act as analgesics in bone cancer pain in mice," Neuroreport, vol. 14, no. 6, pp. 867-869, 2003.

[10] N. M. Luger, M. A. C. Sabino, M. J. Schwei et al., "Efficacy of systemic morphine suggests a fundamental difference in the mechanisms that generate bone cancer vs. inflammatory pain," Pain, vol. 99, no. 3, pp. 397-406, 2002.

[11] H. Vermeirsch, R. M. Nuydens, P. L. Salmon, and T. F. Meert, "Bone cancer pain model in mice: evaluation of pain behavior, bone destruction and morphine sensitivity," Pharmacology Biochemistry and Behavior, vol. 79, no. 2, pp. 243-251, 2004.

[12] P. Reis-Pina, P. G. Lawlor, and A. Barbosa, "Adequacy of cancer-related pain management and predictors of undertreatment at referral to a pain clinic," Journal of Pain Research, vol. 10, pp. 2097-2107, 2017. 
[13] K. Y. Ho, J. S. Ahn, M. M. Calimag et al., "Inadequate treatment practices for pain relief and adverse event management in cancer patients across 10 countries/regions in Asia: a call for greater efforts to improve standards for patient care," Asia-Pacific Journal of Clinical Oncology, vol. 14, no. 3, pp. 159-166, 2018.

[14] H. Breivik, N. Cherny, B. Collett et al., "Cancer-related pain: a pan-European survey of prevalence, treatment, and patient attitudes," Annals of Oncology, vol. 20, no. 8, pp. 1420-1433, 2009.

[15] L. Xu, Y. Wan, J. Huang, and F. Xu, "Clinical analysis of electroacupuncture and multiple acupoint stimulation in relieving cancer pain in patients with advanced hepatocellular carcinoma," Journal of Cancer Research and Therapeutics, vol. 14, no. 1, pp. 99-102, 2018.

[16] W. Dang and J. Yang, "Clinical study on acupuncture treatment of stomach carcinoma pain," Journal of Traditional Chinese Medicine, vol. 18, no. 1, pp. 31-38, 1998.

[17] W. Lu, U. A. Matulonis, J. E. Dunn et al., "The feasibility and effects of acupuncture on quality of life scores during chemotherapy in ovarian cancer: results from a pilot, randomized sham-controlled trial," Medical Acupuncture, vol. 24, no. 4, pp. 233-240, 2012.

[18] H. Chen, T.-Y. Liu, L. Kuai, J. Zhu, C.-J. Wu, and L.-M. Liu, "Electroacupuncture treatment for pancreatic cancer pain: a randomized controlled trial," Pancreatology, vol. 13, no. 6, pp. 594-597, 2013.

[19] D. L. Hershman, J. M. Unger, H. Greenlee et al., "Effect of acupuncture vs sham acupuncture or waitlist control on joint pain related to aromatase inhibitors among women with early-stage breast cancer," JAMA, vol. 320, no. 2, pp. 167-176, 2018.

[20] M. Y. Qiliang, Involvement of Spinal Glial Cells in Animal Model of Cancer Pain and Electroacupuncture Analgesia Observation, Vol. 129, Fudan University, Shanghai, China, 2007.

[21] R.-X. Zhang, A. Li, B. Liu et al., "Electroacupuncture attenuates bone cancer pain and inhibits spinal interleukin- $1 \beta$ expression in a rat model," Anesthesia \& Analgesia, vol. 105, no. 5, pp. 1482-1488, 2007.

[22] M. Y. Qiliang, D. Ren, W.-1. Mi, Q. Liu, and Y. Wang, "Analgesic effects of electroacupuncture combined with Celebrex on rats with tibial cancer pain," Journal of Chinese Integrative Medicine, vol. 6, no. 8, pp. 830-835, 2008.

[23] R.-X. Zhang, A. Li, B. Liu et al., "Electroacupuncture attenuates bone cancer-induced hyperalgesia and inhibits spinal preprodynorphin expression in a rat model," European Journal of Pain, vol. 12, no. 7, pp. 870-878, 2008.

[24] X. Li, Role of the Spinal Toll-like Receptor 4 in Tibia Cancer Pain in Rat and the Anti-allodynic Effect of Electroacupuncture with Different Stimulation Parameters, Vol. 96, Fudan University, Shanghai, China, 2010.

[25] B. A. Smeester, M. Al-Gizawiy, and A. J. Beitz, "Effects of different electroacupuncture scheduling regimens on murine bone tumor-induced hyperalgesia: sex differences and role of inflammation," Evidence-Based Complementary and Alternative Medicine, vol. 2012, Article ID 671386, 16 pages, 2012.

[26] L. Kuai, H. Chen, T. T. Zhang, and Y. H. Yang, "Study on dose-effect relationship of electroacupunctue with different current intensities alleviating tibial cancer pain and inhibition of expression of spinal GFAP in rats," Chinese Acupuncture \& Moxibustion, vol. 32, no. 4, Article ID 2012358462, 2012.

[27] L. Si-ma, B. t. Liu, J. C. Li, N. G. Liu, and Y. Zhang, "Effect of electroacupuncture treatment on pain behavior and calcitonin-gene related peptide expression in cancer induced bone pain-morphine tolerance rat model," Chinese Journal of Behavioral Medicine and Brain Science, vol. 22, no. 5, Article ID 2013441557, 2013.

[28] W. L. Zhao, W. S. Zhao, H. W. Huang, and L. Zhong, "Effects of electroacupuncture on the hypothalamic $\beta$ - endorphin in rats with bne cancer pain," Journal of Clinical Acupuncture Medicine, vol. 29, no. 5, pp. 69-72+91, 2013.

[29] W. L. Zhao, "Experimental Research of electroacupuncture intervene rats of bone cancer pain," HeiLongJiang University of Chinese Medicine, vol. 97, 2013.

[30] J. Y. Du, C. Y. T Chen, S. F. Wu, J. F. Fang, Y. Liang, and J. Q. Fang, "Screen the parameter of electroacupuncture's analgesic after effect on bone cancer pain," CJTCMP, vol. 29, no. 05, pp. 1454-1458, 2014.

[31] J. Y. Du, J. F. Fang, Y. T. Chen, S. F. Wu, Y. Liang, and J. Q. gn Fang, "Parametric optimization of electroacupuncture against bone-cancer pain in rats and its intervention on mRNA expression of opioid receptor and precursor," Chinese Acupuncture \& Moxibustion, vol. 35, no. 2, pp. 161-168, 2015.

[32] Q. B. Lu, F. Wang, T. T. Zhang, L. Kuai, and H. Chen, "Effects of electroacupuncture with different current intensities on $\beta$-endorphin levels in peripheral blood in rats with tibial cancer pain," CJTCMP, vol. 30, no. 9, Article ID 2015800205, 2015.

[33] Q. B. Lu, H. Chen, G. W. Cui, F. Wang, T. T. Zhang, and L. Kuai, "Effects of electroacupuncture and morphine on hyperalgesia and activation of spina astrocyte in rats with bone cancer pain," Shanghai Journal of Traditional Chinese Medicine, vol. 50, no. 5, pp. 77-82, 2016.

[34] L. Sima, B. F. Fan, L. T. Yan, and Y. Shui, "Effects of electroacupuncture treatment on bone cancer pain model with morphine tolerance," Evidence-Based Complementary and Alternative Medicine, vol. 2016, Article ID 8028474, 5 pages, 2016.

[35] T. F. Fu, J. Y. Du, Y. T. Chen, J. F. Fang, Y. Liang, and J. Q. Fang, "Analgesia effect of electroacupuncture at different time on morphine tolerance in rats with bone cancer pain," Journal of ZheJiang Chinese Medical University, vol. 41, no. 6, pp. 447-453, 2017.

[36] T. F. Fu, L. L. Wang, J. Y. Du et al., "Effects of electroacupuncture on expression of $\mu$-opioid receptor in nucleus ceruleus in rats with bone cancer pain-morphine tolerance," Chinese Acupuncture \& Moxibustion, vol. 37, no. 5, pp. 513-520, 2017.

[37] J. F. Guan, B. Wang, S. Wang, Z. W. Hu, and H. Yang, "Effects of electroacupuncture combined with herbal plaster on TLR9 and TLR9 mRNA in spinal dorsal horn of rats with bone cancer pain," Chinese Journal of Gerontology, vol. 38, no. 18, pp. 4513-4516, 2018.

[38] Y. Liang, J.-Y. Du, J.-F. Fang et al., “Alleviating mechanical allodynia and modulating cellular immunity contribute to electroacupuncture's dual effect on bone cancer pain," Integrative Cancer Therapies, vol. 17, no. 2, pp. 401-410, 2018.

[39] Y. Liang, J. Y. Du, J. F. Fang et al., "Analgesic effect of electroacupuncture and its intervention on peripheral opioid receptor expression in rats with bone cancer pain," CJTCMP, vol. 33, no. 11, pp. 4877-4882, 2018.

[40] S. L. Qin, Y. G. Wu, J. F. Guan, and Q. Zhang, "Study on analgesic effect of electroacupuncture combined with pain sticker on rats with bone cancer pain based on reduction of TLR9 expression," Chongqing Medicine, vol. 48, no. 3, pp. 390-393, 2019. 
[41] Y. Q. Cai, J. Y. Du, J. F. Fang et al., "Effect of electroacupuncture on pain threshold and splenic NK cells in rats with bone cancer pain induced by Walker 256 mammary gland carcinoma cells," Chinese Archives of Traditional Chinese Medicine, vol. 37, no. 1, pp. 137-141, 2019.

[42] R. Y. Shi, T. F. Fu, Y. Q. Cai et al., "Electroacupuncture intervention relieves pain possibly by promoting MOR endocytosis in locus coeruleus in bone cancer pain rats with morphine tolerance," Acupuncture Research, vol. 44, no. 3, pp. 161-169, 2019.

[43] J. F. Guan, S. Wang, Z. W. Hu, and B. Wang, "Analgesic effect and possible mechanism of electroacupuncture combined with herbal plaster on rats with cancer bone pain," Journal of Preventive Medicine Chinese PLA, vol. 37, no. 3, pp. 154-155, 2019.

[44] H. MacPherson, D. G. Altman, R. Hammerschlag et al., "Revised STandards for reporting interventions in clinical trials of acupuncture (STRICTA): extending the CONSORT statement," PLoS Medicine, vol. 7, no. 6, Article ID e1000261, 2010.

[45] C. R. Hooijmans, M. M. Rovers, R. B. de Vries, M. Leenaars, M. Ritskes-Hoitinga, and M. W. Langendam, "SYRCLE's risk of bias tool for animal studies," BMC Medical Research Methodology, vol. 14, p. 43, 2014. 